\title{
Logical Intuition Is Not Really About Logic
}

Omid Ghasemi ${ }^{1}$, Simon Handley ${ }^{1}$, Stephanie Howarth ${ }^{1}$, Ian R. Newman ${ }^{2}$, Valerie A. Thompson ${ }^{2}$

${ }^{1}$ Department of Cognitive Science, Macquarie University, Sydney, Australia

${ }^{2}$ Department of Psychology, University of Saskatchewan, Saskatoon, Canada

(C) 2021, American Psychological Association. This paper is not the copy of record and may not exactly replicate the authoritative document published in the APA journal. The final article is available, upon publication, at DOI: 10.1037/xge0001179

Corresponding author:

Omid Ghasemi (Macquarie University, Sydney, NSW, 2109, AUSTRALIA)

Email: omidreza.ghasemi@hdr.mq.edu.au

This research was supported by the Macquarie University Research Excellence Scholarship (iMQRES, Allocation No. 2018459/20191525) Which was awarded to OG., and by a Discovery Grant from the Natural Science and Engineering Research Council of Canada to VT. Online materials, analysis codes, and data are accessible via the Open Science Framework website (https://osf.io/mfhsq/). The first two experiments were presented in the Australian Experimental Psychology Conference (EPC). 


\begin{abstract}
Recent research suggests that reasoners are able to draw simple logical or probabilistic inferences relatively intuitively and automatically, a capacity which has been termed "logical intuition" (see, for example, De Neys \& Pennycook, 2019). A key finding in support of this interpretation is that conclusion validity consistently interferes with judgments of conclusion believability, suggesting that information about logical validity is available quickly enough to interfere with belief judgments. In this paper we examined whether logical intuitions arise because reasoners are sensitive to the logical features of problem or another structural feature that just happens to aligns with logical validity. In three experiments $(N=113,137$, and 254), we presented participants with logical (determinate) and pseudological (indeterminate) arguments and asked them to judge the validity or believability of the conclusion. Logical arguments had determinately valid or invalid conclusions, whereas pseudo-logical arguments were all logically indeterminate, but some were pseudo-valid (possible 'strong' arguments) and others pseudo-invalid (possible 'weak' arguments). Experiments 1 and 2 used simple Modus Ponens and Affirming the Consequent structures; Experiment 3 used more complex Denying the Antecedent and Modus Tollens structures. In all three experiments, we found that pseudo-validity interfered with belief judgments to the same extent as real validity. Altogether, these findings suggest that whilst people are able to draw inferences intuitively, and these inferences impact on belief judgments, they are not 'logical intuitions.' Rather, the intuitive inferences are driven by the processing of more superficial structural features that happen to align with logical validity.
\end{abstract}

Keywords: Reasoning; Dual process theory; Logical intuition; Individual differences; Conflict Detection 


\section{Introduction}

Recent research in the field of reasoning and decision-making suggests that people are able to detect and produce normatively correct responses quite automatically and intuitively (Bago \& De Neys, 2019; De Neys \& Pennycook, 2019; Newman et al., 2017; Pennycook et al., 2015; Trippas et al., 2017). This automatic capacity, which has been called "logical intuition" or "intuitive logic", contradicts traditional theories of reasoning, according to which, intuition relies on one's gut feelings and has no access to logical, causal, or probabilistic norms (Morewedge \& Kahneman, 2010; Sloman, 1996; but see, Pennycook et al., 2018). Whilst this capacity has been labelled "logical intuition" (De Neys, 2014; Handley \& Trippas, 2015; Thompson \& Newman, 2017), to date, there has been no evidence supplied to support the contention that logic (or probability or other normative rules), produces the phenomenon in question. Indeed, it is possible that people are exploiting a structure of the problem that is correlated with properties such as validity, rather than computing answers based on validity per se. In this paper, we test the hypothesis that, in the case of deductive logic, reasoners rely on structural features that happen to align with logical validity in their intuitive judgments rather than computing answers based on actual logic.

Dual process theories make a distinction between type 1, intuitive processes and type 2 , deliberative processes (Epstein, 1994; Evans \& Stanovich, 2013; Sloman, 2012). Based on the traditional version of this theory (Evans, 2010; Evans, 2008), deliberative processes rely on abstract, decontextualized thinking in accordance with rules. These rules, whether they be binary logic rules or causal and probabilistic rules (Evans et al., 2015; Singmann et al., 2016), are assumed to be mainly, if not exclusively, accessible by type 2 thinking (Sloman, 1996; but see, Evans, 2017; Sloman, 2012; Stanovich, 2018). On the other hand, intuitive thinking is attributed to processing features of a problem irrelevant to normative rules (Klauer et al., 2000; Tversky \& Kahneman, 1983). Theorists assume that intuitive processes cause reasoning failures by overreliance on such irrelevant features instead of more relevant ones. For example, in belief bias, which is one of the most widely studied biases in the field, 
reasoners evaluate an argument based on the believability of its conclusion rather than its logical validity.

This view has been recently challenged by new findings indicating that intuitive thinking can draw on logical and probabilistic rules, producing normatively correct responses without engagement of deliberative thinking (De Neys, 2014; Handley et al., 2016; Handley \& Trippas, 2015; Howarth et al., 2021; Pennycook et al., 2015; Thompson \& Newman, 2017). This effect has been investigated with several different paradigms (see Table $\mathrm{S} 1$ in the online materials for an overview of these paradigms). In the conflict detection paradigm (De Neys, 2012) participants are presented with conflict and nonconflict reasoning arguments. On conflict arguments, logical validity is at odds with the conclusion's believability (e.g., valid-unbelievable and invalid-believable arguments). On non-conflict arguments, on the other hand, logical validity aligns with the conclusion's believability (e.g., valid-believable and invalid-unbelievable arguments). Examples of each problem type can be found in Table 1. Researchers have found that reasoners are less confident and slower in their responses to conflict arguments compared to non-conflict ones, irrespective of whether a biased or a logical response is given (De Neys \& Glumicic, 2008; Frey et al., 2017; Šrol \& De Neys, 2020; Thompson \& Johnson, 2014). This effect remains significant even when the chance of engagement in analytical thinking is minimised through the implementation of response deadlines or by increasing cognitive load (Bago \& De Neys, 2017, 2020). This effect is difficult to explain in the traditional dual process accounts, because a slow response (based on logic) should not be able to interfere with a quick one (based on believability), and hence, reasoners should not respond differently to conflict and non-conflict items. The interference effects suggest that reasoners have an intuitive and automatic access to logical and probabilistic rules.

The two-response paradigm is another method used to examine the so-called intuitive sensitivity to logical validity and probability (Thompson et al., 2011; Thompson \& Johnson, 2014). In this paradigm, participants are presented with a problem twice, once under cognitive load and response deadline (Time 1) and once under no load or deadline manipulations (Time 2). Newman, Gibb, and Thompson (2017) demonstrated that participants are able to draw rule-based inferences quickly and intuitively, as 
indicated by a sensitivity to probability and logic rules at Time 1 . Another method to examine intuitive logic in the two-response paradigm is to analyse the direction of response change from Time 1 to Time 2. According to the traditional dual process theorists, one main role of analytical thinking is to evaluate the justifiability of initial responses and to correct, when necessary, biased intuitive responses (Evans, 2017; Kahneman \& Frederick, 2002). Contrary to the corrective hypothesis of dual process theory, direction of change analysis has shown that the majority of normatively correct responses are produced at Time 1 rather than corrected at Time 2 (Bago \& De Neys, 2019; Raoelison et al., 2020).

Another line of evidence in support of logical intuitions comes from studies that have shown people are sensitive to logical validity even on tasks in which the required response is unrelated to logical structure. One such method draws upon the misattribution paradigm (Topolinski, 2018; Topolinski \& Reber, 2010). Researchers in the field of reasoning and judgment have adapted this paradigm by asking participants to judge the likability and physical brightness of the concluding statement to logical arguments. Based on these instructions, the logical structure of the argument is an irrelevant feature and should not influence responses. However, the results have shown that liking and brightness judgments are higher for valid compared to invalid arguments (Ghasemi, Handley, \& Howarth, 2021; Morsanyi \& Handley, 2012; Trippas et al., 2016). According to the misattribution paradigm, upon reading a coherent valid argument, a sense of fluency is triggered, which in turn, leads to positive affect; this positive feeling is then misattributed to judgments of liking and physical brightness. It has been argued that these findings are consistent with the claim that participants are automatically processing logical structure, which interferes with their ability to make judgments based upon the relevant characteristics of the task. However, more recent research suggests that the effect of logic on liking ratings may be less intuitive than originally thought, since they can be affected by manipulations (e.g., availability of time and cognitive resources) that hinder deliberative thinking (Hayes et al., 2020; Nakamura \& Kawaguchi, 2016). Brightness judgments, on the other hand, are not affected by such manipulations (Ghasemi, Handley, \& Howarth, 2021). 
Another paradigm in which logical validity interferes with an unrelated judgment is the instructional manipulation paradigm (Handley et al., 2011). In a typical instructional manipulation study, participants are instructed to evaluate conflict and non-conflict reasoning problems either based on logical rules (i.e., LOGIC instructions) or the believability of their conclusions (i.e., BELIEF instructions). The difference in accuracy between conflict and non-conflict items under belief instructions is assumed to be an indicator of intuitive sensitivity to logic, whilst the the effect of conflict under logic instructions is assumed to be an indicator of intuitive beliefs. Studies with such methods have demonstrated that the effect of conflict for simpler reasoning arguments is at least as large under belief instructions as it is under logic instructions (Handley et al., 2011; Howarth et al., 2018; Ricco et al., 2020; Trippas et al., 2017). In other words, the logical validity of an argument interferes with making belief judgments, presumably because the intuitive sensitivity to logical validity interferes with judgments of believability (Trippas \& Handley, 2018). Similarly, Pennycook et al. (2014) used base rate problems and asked participants to respond either based on statistical information or stereotype information. They found a similar pattern in which base-rate information interfered with peoples beliefs about how well a profession aligned with a presented description. Collectively, these results seem to indicate that reasoners process the logical validity of an argument quickly and intuitively enough that it interferes with making belief-based inferences.

\subsection{Underlying Mechanisms of Logical Intuition}

Four separate lines of research converge on the same conclusion, namely that reasoners are intuitively sensitive to the logical structure of inferences. However, a key question concerns how such logical intuitions arise. One possibility is that during formal education as well as in everyday situations, simple logical structures (e.g., if it snows, the match will be cancelled, it snowed, therefore the match was cancelled) are used and practiced to the extent that people can draw such inferences quite automatically (Stanovich, 2018). Although early evidence shows that developmental increases in biased responding are associated with increases in working memory capacity (Morsanyi \& Handley, 2008), the recent finding that children, as they get older, not only get better at correcting their initial incorrect responses, but also at producing the initial correct response without any engagement in deliberative 
reasoning (Raoelison et al., 2021) supports this automatization process. Alternatively, perhaps these simple inferences (such as modus ponens or disjunction elimination) are part of our fundamental cognitive architecture, the sort of simple 'direct' rules of inference often included in the natural deduction systems described by rule based theorists of reasoning (Braine \& O'Brien, 1998; Rips, 1994). In either case, the argument is similar: reasoners are equipped with an intuitive capacity to detect logical inferences and distinguish conclusions that are logically valid from those that are not.

In this paper we consider an alternative possibility, namely that the effects of 'intuitive logic' may have nothing to do with logical validity per se, but may instead reflect sensitivity to some other structural feature of the problem that just happens to align with logical validity. Our experimental approach makes use of common conditional argument forms such as modus ponens (MP) and affirmation of the consequent (AC). Whilst MP arguments are either logically valid or invalid, AC arguments are logically indeterminate, although they are regularly endorsed as valid by many reasoners (Evans et al., 2007). Consider the following example of the AC argument:

If Camilla eats an ice lolly then her mouth will get cold

Camilla's mouth got cold

Therefore, Camilla ate an ice lolly

Whilst the conclusion is possible given the premises, it does not necessarily follow from the premises. The conditional premise indicates that ice lollies are always associated with cold mouths, but is silent on the reverse inference; thus, the fact of a cold mouth does not licence an inference about its source. Indeed, it is easy to imagine alternative reasons that Camilla's mouth got cold (such as drinking 
a cold drink). Thus, the concusion is possible given the premises, but it is not necessitated by the premises $^{1}$.

In this paper, we investigate whether invalid, but plausible, inferences, such as $\mathrm{AC}$, are intuitively available in a similar way to valid inferences, such as MP. If we were to observe 'intuitive logic' effects with $\mathrm{AC}$, then it would demonstrate that these effects cannot be explained by intuitive sensitivity to normative logical rules. Instead it might suggest that they are driven by the processing of more superficial structural features. We will return to candidates for these structural features in the general discussion.

\subsection{The Present Study}

The goal of the present study was to evaluate whether reasoners are genuinely sensitive to logical validity in their intuitive judgments, or rather, swayed by more superficial problem features that may be aligned with logical validity. To test this hypothesis, we employed the instructional manipulation paradigm introduced earlier, in which participants are asked to make judgments based upon the believability or logical validity of the conclusion. Recall that, on problems in which there is a conflict between beliefs and logic, belief judgments tend to take longer and result in more errors than when there is no conflict. This finding has been argued as strong evidence for logical intuition, as it shows that information about logical validity is available early and interferes with judgments of conclusion believability. In addition to presenting problems that had logically valid or invalid conclusions, we gave reasoners problems where there was a plausible or implausible conclusion that was in conflict with beliefs. In other words, we gave people a structure they could potentially exploit that was not derived

${ }^{1}$ Of course, the conclusion to an AC argument is valid under a biconditional reading of the conditional premise. However, the general pattern of reasoning observed from conditional arguments is inconsistent with a biconditional reading. We will return to this issue in general discussion 
from logical validity, and we sought to know whether they would exploit it. Consider, for example, the following two arguments:

A) If the child is laughing then she is sad The child is sad Therefore the child is laughing

B) If the child is laughing then she is sad The child is sad Therefore the child is crying

Both of these are AC arguments, and thus, both have the same logical status: they are both invalid. However, it is clear that they are not equally appealling: argument A appears to be stronger than argument B. We will refer to arguments such as A) as 'pseudo valid' because they have a logical form corresponding to a typical $\mathrm{AC}$ argument (if $p$ then $q$, $q$, therefore $p$ ). We will refer to arguments such as B) as 'pseudo invalid' (if $p$ then $q$, $q$, therefore not- $p$ ). Studies have demonstrated that participants usually endorse pseudo-valid (i.e., possible strong) arguments as valid and reject pseudo-invalid (i.e., possible weak) arguments as invalid (Evans et al., 2001; Evans et al., 1999). Both A) and B) are conflict arguments in so far as the believability of the conclusion is in conflict with the 'pseudo validity' of the conclusion. For example, if reasoners are asked to judge the believability of the conclusion in A), the response should be 'No' because the conclusion is not believable; however this choice would conflict with the tendency to draw the $\mathrm{AC}$ inference (that the child is laughing). In contrast, the conclusion to argument B) is believable so one would expect reasoners to answer 'Yes' but, unlike argument A, B is 'pseudo invalid' and invites rejection.

The primary goal of this study is to determine whether "pseudo validity" interferes with judgments of conclusion believability in a manner similar to that observed with determinately valid and invalid arguments. If this is the case, then reasoners' responses to the "pseudo valid" arguments would mirror the responses observed on determinately valid arguments. Such an observation would suggest that the source of conflict in the instruction paradigm is not logical validity per se, given that interference can arise from non-logical structures. On the other hand, if the phenomenon known as "logical" intuition really does rely on logic, then we should only observe interference effects when we manipulate logical validity, and not when we manipulated pseudo-validity. 
In the current paper, we used the instructional manipulation paradigm across three experiments. In Experiment 1, we used the paradigm described above, varying validity/ pseudo validity orthogonally to conclusion believality. In Experiment 2, we used the same materials and design, but presented participants with two measures of cognitive capacity. Whilst several studies have showed that reasoners with higher cognitive capacities have increased intuitive sensitivity to logical validity (Erceg et al., 2019; Frey et al., 2017; Raoelison et al., 2020; Šrol \& De Neys, 2020; Thompson et al., 2018; Thompson \& Johnson, 2014), others have failed to find such a relationship between logical intuition capacity and cognitive ability (Ghasemi, Handley, \& Howarth, 2021; Markovits et al., 2020; Morsanyi \& Handley, 2012; Nakamura \& Kawaguchi, 2016). The goal of this experiment was to test whether higher ability participants are similarly sensitive to both logic and pseudo-logic structures. Finally, in Experiment 3A, we extended our analysis to include modus tollens (MT) and denial of the antecedent (DA) conditional arguments in order to test if a simple matching heuristic could explain the results of Experiments 1 and

2. Moreover, MT and DA arguments are considered as more complex inferences than MP and AC (Ricco et al., 2020; Schaeken \& Schroyens, 2000; Schroyens et al., 2000, 2001; Schroyens \& Braem, 2011; Trippas et al., 2017). Thus, another goal of the third experiment is to examine whether the effects extended to more complex inferences.

\section{Experiment 1}

In Experiment 1, participants were presented with modus ponens (MP) and affirmation of the consequent (AC) argument forms. MP arguments were determinate with valid and invalid forms, whilst all AC arguments were indeterminate. However, as discussed above, participants usually endorse one form of these arguments as valid and the other form as invalid (Evans et al., 1999). Thus, we labelled these two forms as pseudo-valid and pseudo-invalid, respectively. As in previous studies, we created conflict and non-conflict arguments by crossing conclusion believability with the (pseudo-) logical validity of the arguments.

The experimental task used an instructional manipulation which required participants to evaluate an argument's conclusion based on an instructional cue that appears shortly after the first two premises. 
In other words, participants were instructed to judge whether the conclusion necessarily follows the premises (i.e., logic instructions) or whether the conclusion is believable or unbelievable (i.e., belief instructions).

Under logic instructions, the difference in accuracy on conflict and non-conflict items corresponds to the "belief bias" effect (Evans et al., 1999; Klauer et al., 2000; Newstead et al., 1992). Conversely, the effect of conflict under belief instructions indicates the interference of logical structure on belief judgments. This interference is evident in lower accuracy, longer response times, and lower confidence on conflict arguments compared to non-conflict arguments (Thompson et al., 2018; Trippas et al., 2017). Thus, in the instructional manipulation paradigm, logical intuition is defined as the effect of conflict under belief instructions (Trippas \& Handley, 2018). If "logical intuition" is sensitive to logical validity, we expect to see different patterns in responding to logical and pseudo-logical arguments: A genuine logical intuition that is sensitive to normative logical rules would manifest as an effect of conflict on MP logical arguments, but not on AC arguments while assessing the believability of arguments. On the other hand, a similar pattern of conflict on the two argument types would suggest that logical intuition is not relying on the underlying logical structure of arguments, but rather on some other regularity in the problem.

\subsection{Method}

\subsubsection{Ethics Statement}

All experiments of this study were approved by the Macquarie University Human Science Ethics Committee (Reference Number: 26289).

\subsubsection{Participants}

One hundred and thirteen students from the University of Saskatchewan participated in this experiment ( 75 were female, $M$ age $=20.78, S D=3.48$ ). All participants received one course credit to take part in the experiment. 


\subsubsection{Materials}

In this experiment, participants were presented with 32 modus ponens (MP) and 32 affirmation of the consequent (AC) conditional arguments. For each type, half of the arguments were conflict problems and the other half were non-conflict problems. For MP arguments, the conflict was between the logical status and believability of the argument. Conflict problems were valid-unbelievable and invalid-believable arguments, and non-conflict problems were valid-believable and invalidunbelievable arguments. Valid problems had the "If $p$ then $q$; $p$; therefore $q$ " form and invalid problems had the "If $p$ then $q$; $p$; therefore, not $q$ " form.

For AC arguments, the conflict was between the pseudo-validity and believability of the argument. Conflict problems included pseudo-valid-unbelievable (i.e., possible strong unbelievable) and pseudoinvalid-believable (i.e., possible weak believable) arguments, and non-conflict problems included pseudo-valid-believable (i.e., possible strong believable) and pseudo-invalid-unbelievable (i.e., possible weak unbelievable) arguments. We used 8 of each argument type in the conflict and nonconflict categories. Pseudo-valid problems were of the form of "If $p$ then $q$; $q$; therefore, $p$ " and pseudoinvalid problems were in the form of "If $p$ then $q$; q; therefore, not $p$ ". For the ease of presentation, we refer to all MP arguments as "Logical Arguments" and all AC arguments as "Pseudo-Logical Arguments". An example of each argument type can be found in Table 1.

Table 1: Examples of argument types across conflict conditions for experiments 1 to 3.

\begin{tabular}{|c|c|c|c|c|}
\hline & \multicolumn{2}{|c|}{ Non-conflict } & \multicolumn{2}{|c|}{ Conflict } \\
\hline & Valid/Believable & Invalid/Unbelievable & Valid/Unbelievable & Invalid/Believable \\
\hline $\begin{array}{l}\text { Modus } \\
\text { ponens }\end{array}$ & $\begin{array}{l}\text { P1: If John is in the water [p] } \\
\text { then he is wet [q] } \\
\text { P2: John is in the water [p] } \\
\text { C: John is wet [q] }\end{array}$ & $\begin{array}{l}\text { P1: If John is in the water } \\
{[\mathrm{p}] \text { then he is wet }[\mathrm{q}]} \\
\mathrm{P} 2: \text { John is in the water [p] } \\
\text { C: John is dry }[\neg \mathrm{q}]\end{array}$ & $\begin{array}{l}\text { P1: If John is in the water } \\
\text { [p] then he is dry [q] } \\
\text { P2: John is in the water } \\
\text { [p] } \\
\text { C: John is dry [q] }\end{array}$ & $\begin{array}{l}\text { P1: If John is in the water } \\
\text { [p] then he is dry [q] } \\
\text { P2: John is in the water [p] } \\
\text { C: John is wet }[\neg q]\end{array}$ \\
\hline $\begin{array}{l}\text { Modus } \\
\text { tollens }\end{array}$ & $\begin{array}{l}\text { P1: If John is out of the water } \\
\text { [p] then he is dry [q] } \\
\text { P2: John is wet }[\neg q] \\
\text { C: John is in the water }[\neg p]\end{array}$ & $\begin{array}{l}\text { P1: If John is out of the } \\
\text { water [p] then he is dry [q] } \\
\text { P2: John is wet }[\neg q] \\
\text { C: John is out of the water } \\
\text { [p] }\end{array}$ & $\begin{array}{l}\text { P1: If John is in the water } \\
\text { [p] then he is dry [q] } \\
\text { P2: John is wet }[\neg q] \\
\text { C: John is out of the } \\
\text { water }[\neg p]\end{array}$ & $\begin{array}{l}\text { P1: If John is in the water } \\
\text { [p] then he is dry [q] } \\
\text { P2: John is wet }[\neg q] \\
\text { C: John is in the water [p] }\end{array}$ \\
\hline & Pseudo-Valid /Believable & $\begin{array}{l}\text { Pseudo-Invalid } \\
\text { /Unbelievable }\end{array}$ & $\begin{array}{l}\text { Pseudo-Valid } \\
\text { /Unbelievable }\end{array}$ & $\begin{array}{l}\text { Pseudo-Invalid/ } \\
\text { Believable }\end{array}$ \\
\hline $\begin{array}{l}\text { Affirmation } \\
\text { of the } \\
\text { consequent }\end{array}$ & $\begin{array}{l}\text { P1: If John is out of the water } \\
\text { [p] then he is dry [q] } \\
\text { P2: John is dry [q] } \\
\text { C: John is out of the water [p] }\end{array}$ & $\begin{array}{l}\text { P1: If John is out of the } \\
\text { water [p] then he is dry [q] } \\
\mathrm{P} 2: \text { John is dry [q] } \\
\text { C: John is in the water [ } \neg \mathrm{p}]\end{array}$ & $\begin{array}{l}\text { P1: If John is in the water } \\
\text { [p] then he is dry [q] } \\
\text { P2: John is dry [q] } \\
\text { C: John is in the water } \\
\text { [p] }\end{array}$ & $\begin{array}{l}\text { P1: If John is in the water } \\
\text { [p] then he is dry [q] } \\
\text { P2: John is dry [q] } \\
\text { C: John is out of the water } \\
{[\neg \text { p] }}\end{array}$ \\
\hline
\end{tabular}




\begin{tabular}{|c|c|c|c|c|}
\hline $\begin{array}{l}\text { Denial of } \\
\text { the } \\
\text { antecedent }\end{array}$ & $\begin{array}{l}\text { P1: If John is in the water [p] } \\
\text { then he is wet [q] } \\
\text { P2: John is out of the water } \\
{[\neg p]} \\
\text { C: John is dry }[\neg q]\end{array}$ & $\begin{array}{l}\text { P1: If John is in the water } \\
{[\mathrm{p}] \text { then he is wet }[\mathrm{q}]} \\
\mathrm{P} 2: \text { John is out of the water } \\
{[\neg \mathrm{p}]} \\
\text { C: John is wet }[\mathrm{q}]\end{array}$ & $\begin{array}{l}\text { P1: If John is in the water } \\
\text { [p] then he is dry [q] } \\
\text { P2: John is out of the } \\
\text { water }[\neg p] \\
\text { C: John is wet }[\neg q]\end{array}$ & $\begin{array}{l}\text { P1: If John is in the water } \\
\text { [p] then he is dry [q] } \\
\text { P2: John is out of the } \\
\text { water }[\neg \text { p] } \\
\text { C: John is dry [q] }\end{array}$ \\
\hline
\end{tabular}

Finally, to make sure that the content of each argument did not confound with its logical structure (Klauer \& Singmann, 2013; Singmann et al., 2014), we randomly assigned contents to structures in order to end up with the same number of contents for each argument type and subtype.

\subsubsection{Procedure}

We used PsychoPy3 and PsychoJS library (Peirce et al., 2019) to create the online version of all three experiments. These experiments were hosted on Pavlovia (https://pavlovia.org/). Participants were presented with 64 conditional arguments in a random order and they were instructed to judge either the logical validity or believability of each argument. For logic instructions, they were asked to assume the two premises were true, even if they did not make sense in the real world, and judge whether the conclusion necessarily follows from those premises. For belief instructions, they were instructed to evaluate the conclusion based on what they think is true in the real world. These instructions were adapted from Trippas et al. (2017).

Each trial started with the first two premises on the screen, and after 3 seconds, a conclusion, an instruction cue, and two buttons appeared below a line that separates premises from the conclusion. The instruction cue was either "LOGIC" or "BELIEF" in red, and depending on the cue, the buttons were labelled as either valid-invalid or believable-unbelievable. Participants were instructed to select the appropriate button according to the cue. Following each trial, participants were asked to rate the confidence in their responses. Before starting the main part of the experiment, participants were presented with 8 practice trials to get familiar with the experiment. This experiment lasted for 30 minutes. All the materials and instructions can be found in the online materials at (https://osf.io/mfhsq/). 


\subsection{Results}

\subsubsection{Analysis Approach}

All the analyses were performed using R programming language (R Core Team, 2014). The main hypotheses were tested using the (generalized) linear mixed model from the afex package (Singmann et al., 2015), which is a more suitable method to analyse binary accuracy data and factorial designs than the analysis of variance (Bolker et al., 2009). In order to minimise Type 1 error, we started with the maximal model that is justified by the experimental design (Barr et al., 2013) and simplified the model in case of convergence errors. We performed a stepwise simplification on an overparameterized model by removing the correlation parameter, higher-order interactions, and random effect terms with least variance to address the convergence error (Singmann \& Kellen, 2019). We focused on the accuracy and confidence judgments which were analysed using generalized linear mixed model (GLMM) and linear mixed model, respectively. However, the analyses of confidence ratings were reported briefly in the manuscript but the entire results can be found in the online materials. Moreover, in running post-hoc analyses, which were performed using the emmeans package (Lenth et al., 2018), we used the HolmBonferroni adjustment to correct for multiple comparisons.

In the analysis of accuracy data, we used GLMM with the logit link function. Thus, the model coefficients describe effects on log-odd or latent metrics (Mize, 2019). However, following McCabe et al. (2020) and Mize (2019) for best practices in interpreting non-linear main effects and interactions, all estimates were back-transformed to the natural probability scale. Thus, as you will see in the following sections, it is possible to find a significant main effect or interaction on log-odd scale level but the differences of predicted probabilities are quite small or even zero. Moreover, in addition to the parameter estimated by the model, we interpret all the effects using the predicted probability and the differences of differences in probabilities in the text and figures.

Finally, to make the comparison of the accuracy in both logical and pseudo-logical arguments feasible, we scored the pseudo-valid arguments as correct if they were accepted as valid, and the pseudoinvalid arguments as correct when they were rejected as invalid. This way, we were able to compare responding patterns in both argument types directly. The tables containing estimated mean accuracy 
and confidence ratings can be found in the online materials. All materials, data, and analysis scripts can be retrieved at the Open Science framework (https://osf.io/mfhsq/).

\subsubsection{Conflict and Instructions Effects}

To test the main hypotheses of Experiment 1, we performed a GLMM with a logit link function and a binomial family distribution on the accuracy data. This model included conflict (2: conflict vs. nonconflict), instruction (2: logic vs. belief), and argument type (2: logical vs. pseudo-logical) as fixed factors, and by-participant random intercept, by-participant random slope for conflict and instruction, by-participant correlation parameter, by-item random intercept, and by-item random slope for instruction and argument type. The results of the model, as is shown in Figure 1, demonstrated main effects of conflict, $\chi^{2}(1)=83.82, p<.001$, instruction, $\chi^{2}(1)=6.49, p=.01$, and argument type, $\chi^{2}(1)$ $=4.62, \mathrm{p}=.03$. Participants were more accurate on non-conflict than conflict arguments (.98 vs. .81), on logical than pseudo-logical arguments (.94 vs. .93), and under logic instructions than belief instructions $(.95 \text { vs. .91 })^{2}$.

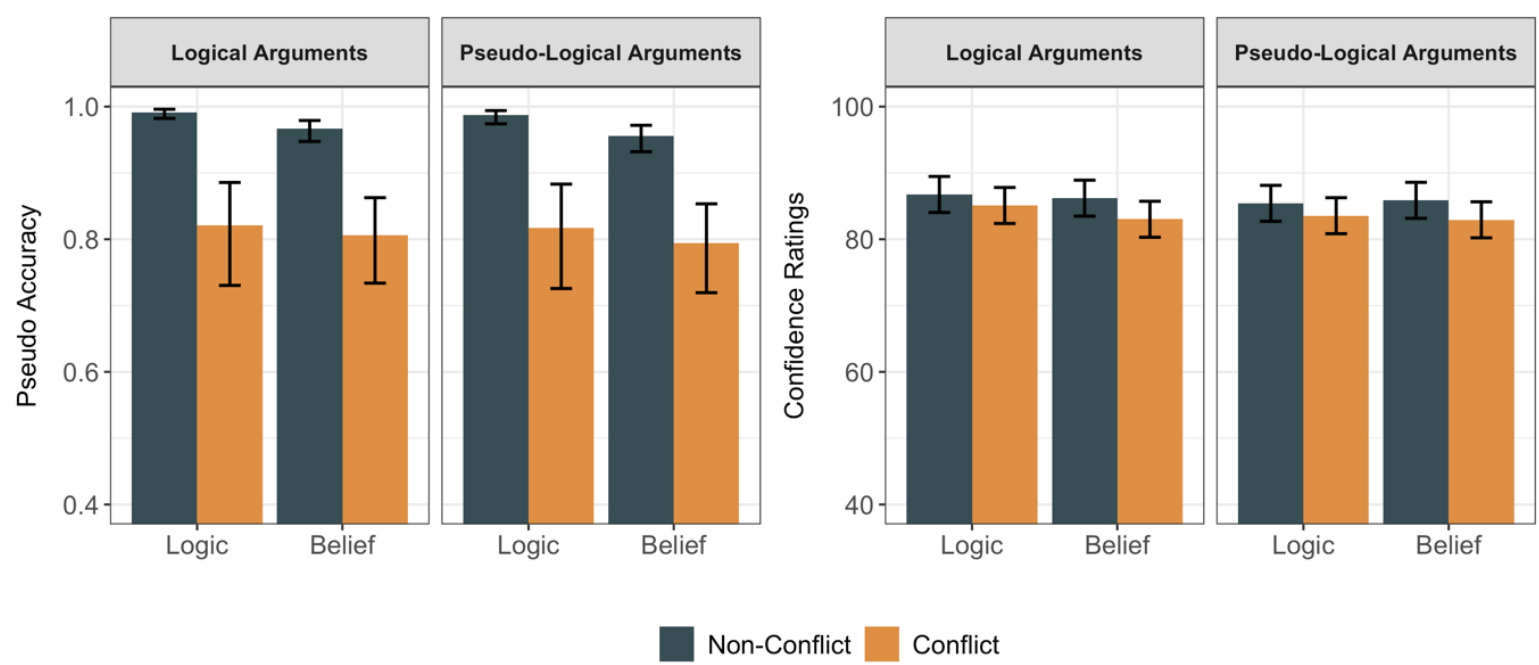

Figure 1: Estimated mean accuracy and confidence ratings for conflict and instruction conditions across logical and pseudo-logical arguments of Experiment 1. Error bars represent $95 \%$ confidence interval.

${ }^{2}$ Considering the way that we coded AC arguments as either pseudo-valid or pseudo-invalid, accuracy reflects the propensity to accept valid and pseudo-valid arguments and reject invalid and pseudo-invalid arguments. However, to make the interpretation of the results easier, we simply report our findings in terms of accuracy instead of endorsement. 


\subsubsection{Pseudo-Intuitive Logic Effect}

The findings revealed a significant conflict by instruction interaction, $\chi^{2}(1)=40.07, p<.001$. Whilst the effect of conflict is significant under both instructional manipulations ( $p s<.001$ ), it was more marked under logic instructions than belief instructions (.17 vs. .14). In contrast with the "logical intuition" account, we hypothesized that conflict interferes with belief judgments for both logical and pseudo-logical arguments. Consistent with this hypothesis, the results showed neither a significant argument type by conflict interaction $\left(\chi^{2}=3.44, p=.06\right)$, nor a three-way argument type by conflict by instruction interaction $\left(\chi^{2}=.25, p=.62\right)$. These findings show that conflict impacted belief judgments equally for valid and pseudo-valid arguments.

\subsubsection{Confidence Ratings}

A linear mixed model with conflict, instruction, and subtype as fixed factors, and by-participant random intercept, by-participant random slope for conflict and instruction, by-item random intercept, and by-item random slope for conflict, instruction, and argument type was performed on confidence ratings. The results indicated an instruction by conflict interaction, $F(1,6878.07)=4.48, p=.03$. Whilst conflict significantly influenced both logic and belief instructions $(p s<.006)$, it interfered more with the latter than the former ( 3.06 vs. 1.76). Consistent with the accuracy data, both the conflict by argument type interaction, and the conflict by argument type by instruction interaction were nonsignificant $(p s>.72)$.

\subsection{Discussion}

The main goal of Experiment 1 was to investigate whether conflict interferes with belief judgments on both logical and pseudo-logical arguments. Consistent with numerous findings in the literature, we found that a conflict between the logical validity and believability of a conclusion affected both accuracy and confidence ratings under both belief and logic instructions (Handley et al., 2011; Howarth et al., 2018; Ricco et al., 2020; Thompson et al., 2018; Trippas et al., 2017): Participants were less accurate and less confident on conflict arguments than non-conflict arguments. Under belief instructions, this sensitivity to conflict has been considered to be evidence for logical intuition (Handley \& Trippas, 2015; Trippas \& Handley, 2018). However, as we predicted, we observed a conflict effect 
of similar magnitude for our pseudo-logical arguments. This finding is consistent with the hypothesis that logical intuitions are not necessarily based on deductive validity. Instead, our findings suggest that conclusion plausibility rather than conclusion necessity underpin the impact of argument structure on belief judgments.

\section{Experiment 2}

The goal of Experiment 2 was to replicate the findings of Experiment 1 and to investigate the relationship between our pseudo-logical conflict effects and cognitive ability. Contrary to expectations, it has been observed that logical intuitions are most likely to be observed amongst high- rather than low-ability reasoners (Erceg et al., 2019; Frey et al., 2017; Hayes et al., 2020; Raoelison et al., 2020; Šrol \& De Neys, 2020; Thompson et al., 2018; Thompson \& Johnson, 2014; but see, Ghasemi, Handley, \& Howarth, 2021; Howarth et al., 2018; Markovits et al., 2020; Nakamura \& Kawaguchi, 2016). The explanation given for this relationship is that high ability reasoners have learned (at least simple) logical inferences to the point of automaticity (Stanovich, 2018; Thompson et al., 2018), so that they interfere with the ability to make belief-based judgement. If this explanation were true, we would expect that the higher ability reasoners would show conflict effects on genuinely valid, but not pseudo-valid problems. By contrast, if high-capacity reasoners are exploiting some structural features of the problems other than logical validity, we might expect them to be more sensitive to the pseudo-logical conflict than lower capacity reasoners.

\subsection{Method}

\subsubsection{Participants}

We recruited 110 students from Macquarie University and 27 students from the University of Saskatchewan (109 were female, $M$ age $=21.56, S D=6.16)$. Participants received two course credits to take part in the experiment. 


\subsubsection{Materials}

We used the same arguments as Experiment 1. In order to measure individual differences, participants were presented with the first part of the AH4 (Heim, 1970), 3-item CRT (Frederick, 2005), and 4-item CRT (Toplak et al., 2014).

\subsubsection{Procedure}

Participants started the experiment by answering individual differences measures. They were presented with all 7 CRT problems in a random order. Following the $12 \mathrm{AH} 4$ example questions, they were given 10 minutes to respond to the first part of the AH4 which includes 65 verbal and quantitative questions. Finally, they were presented with 64 arguments with the same randomization and presentation format as Experiment 1. This experiment lasted for 60 minutes.

\subsection{Results}

The average accuracy on the AH4, CRT-3, and CRT-4 was $39.57(\mathrm{SD}=11.5), .99(\mathrm{SD}=1.15)$, and $1.59(\mathrm{SD}=1.36)$, respectively. The accuracy in these measures were similar to previous experiments with student samples (e.g., Frederick, 2005; Ghasemi, Handley, \& Howarth, 2021; Toplak et al., 2014; Trippas et al., 2013). There were moderate correlations between these measures ( $r s>.32$, ps $<.001)$. As is common practice in previous research on logical intuitions (e.g., Raoelison et al., 2020; Thompson et al., 2018), and also for the ease of presentation, a general cognitive ability test was created by calculating the average z-transformed scores of these measures. This composite measure of cognitive ability had an acceptable reliability $(\alpha=.71)$. We performed a median split on cognitive ability scores and created a low ability group $(n=68)$ and a high ability group $(n=69)$.

Similar to Experiment 1, a GLMM was performed on the accuracy data. This model contained conflict, argument type, instruction, and cognitive ability as fixed factors, and by-participant random intercept, by-participant random slope for conflict and instruction, and a correlation parameter as random factors. All the models with by-item random intercept failed to converge. We begin by considering how the findings align with those reported in Experiment 1 before considering the extent to which ability interacts with these effects. As in Experiment 1, the results showed that participants 
had a higher accuracy on non-conflict than conflict arguments (.98 vs. .86), on logical than pseudological arguments (.95 vs. .94), $\chi^{2}(1)=76.26, p<.001$, and $\chi^{2}(1)=5.39, p=.02$, respectively, and under logic instructions than belief instructions (.97 vs. .92), $\chi^{2}(1)=16.61, p<.001$.

\subsubsection{Pseudo-Intuitive Logic Effect}

We found a marginally significant conflict by instruction interaction, $\chi^{2}(1)=3.79, p=.05$, which, in contrast to Experiment 1, indicated a greater effect of conflict under belief instructions than logic instructions (.15 vs. .09). Most importantly, this two-way interaction was qualified by a higher order three-way interaction of conflict by instruction by argument type, $\chi^{2}(1)=6.81, p=.009$. The pattern of the results in Figure 2 suggests that under belief instructions, the effect of conflict is larger for pseudo-logical arguments than logical ones. This is important because it suggests that the intuitive response generated on pseudo-logical arguments is having a greater impact on belief judgments than the intuitive response generated on logical arguments. We followed up this interaction by running two GLMMs with conflict and argument type as fixed effect factors for logical and pseudo-logical arguments separately. The same simplification procedure was performed on maximal models in case of convergence error.

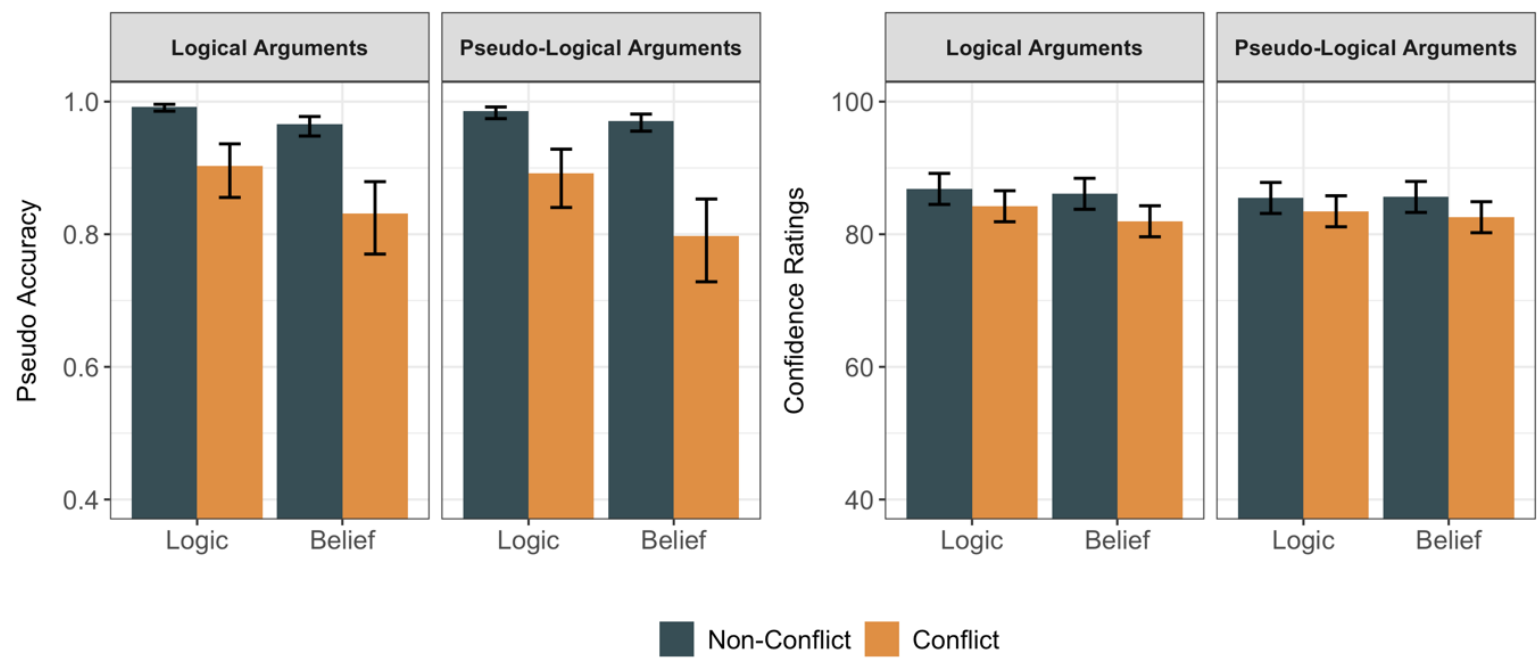

Figure 2: Estimated mean accuracy and confidence ratings for conflict and instruction conditions across logical and pseudo-logical arguments of Experiment 2. Error bars represent $95 \%$ confidence interval.

For the logical arguments, we found a main effect of conflict, $\chi^{2}(1)=75.29, p<.001$, and instruction, $\chi^{2}(1)=11.45, p<.001$, indicating higher accuracy for non-conflict arguments and logic 
instructions. The conflict by instruction interaction was not significant $\left(\chi^{2}=1.60, p=.21\right)$, indicating a similar effect of conflict under belief and logic instructions (.13 vs. .09). For pseudo-logical arguments, on the other hand, the results revealed a main effect of conflict, $\chi^{2}(1)=97.30, p<.001$, and a marginally significant conflict by instruction interaction, $\chi^{2}(1)=3.76, p=.05$. The effect of conflict was larger under belief instructions compared to logic instructions (.18 vs. .11). As in Experiment 1, these findings show that logic significantly interferes with belief judgments, and if anything in Experiment 2, the effect is stronger for pseudo-logical than logical arguments.

\subsubsection{The Effect of Cognitive Ability}

The main analysis also showed a main effect of ability, with higher ability participants showing greater accuracy than low ability participants (.98 vs. .89), $\chi^{2}(1)=27.81, p<.001$. Whilst the GLMM showed that instruction interacted with cognitive ability, $\chi^{2}(1)=6.14, p=.01$, the differences between the two ability groups were significant under both instructions $(p s<.001)$ and the differences when converted back from the log-odd scale to the natural response scale were identical (.08 vs. $\left..08^{3}\right)$. More importantly, this interaction was not qualified with higher order interactions with conflict $\left(\chi^{2}=.21, p=\right.$ $.65)$, argument type $\left(\chi^{2}=3.42, p=.065\right)$, or both $\left(\chi^{2}=.18, p=.67\right)$. These findings show that, contrary to previous research, there is no evidence that higher ability participants are more sensitive to intuitive inferences. Moreover, the pseudo conflict effect did not vary as a function of cognitive ability.

Finally, we note a small instruction by argument type interaction, $\chi^{2}(1)=4.07, p=.04$. Follow-up analyses indicated that the difference between logical and pseudo-logical arguments' accuracy is significant under logic instructions, but not under belief instructions (.01 vs. $.002, p=.01$ and .81 , respectively).

\footnotetext{
${ }^{3}$ As explained previously, although we found an interaction based on the coefficient of the product term, the differences in probabilities
} revealed that such an interaction is absent in the response level. As Mize (2019) and McCabe et al. (2020) suggested, we interpreted interactions in terms of the natural probability scale, rather than transformed log-odd scale of the product term. 


\subsubsection{Confidence Ratings}

A linear mixed model was employed on confidence ratings data. The fixed-effect structure of the model included conflict, instruction, argument type, and cognitive ability. The random-effect structure included by-participant random intercept and slope for conflict and instruction, by-item random intercept, and by-item random slope for conflict, argument type, and cognitive ability. Consistent with Experiment 1 , instruction interacted with conflict, $F(1,8318.93)=5.16, p=.02$, indicating, as is shown in Figure 2, a greater effect of conflict under belief instructions than logic instructions (3.60 vs. 2.31). This interaction was not qualified with a higher order interaction with argument type, $F(1,8328.99)=$ $.18, p=.67$.

\subsection{Discussion}

The results of Experiment 2 revealed that pseudo-logic interfered with belief judgments in the same way that logic did. In other words, in contrast to the notion of "logical intuition as a sensitivity to logic", participants showed intuitive sensitivity to both logic and pseudo-logic. Moreover, the results demonstrated that neither the so-called logical intuition nor pseudo-logical intuition are dependent on cognitive capacities. Contrary to some earlier findings (Erceg et al., 2019; Frey et al., 2017; Raoelison et al., 2020; Ricco et al., 2020; Šrol \& De Neys, 2020; Thompson et al., 2018; Thompson \& Johnson, 2014), we did not find evidence that logical intuitions were more prevalent amongst high-capacity reasoners; and novel to this experiment, we found the interference caused by pseudo conflict also did not vary as a function of cognitive ability.

The lack of a relationship between individual differences in intuitive logic and cognitive ability in the current study is consistent with studies which have mainly used instructional manipulations to measure intuitive inferences. For example, Ghasemi et al. (2021) found that when participants were instructed to evaluate the physical brightness of the conclusions to reasoning arguments, high and low ability participants rated valid arguments as brighter than invalid arguments to similar degrees. Similarly, Howarth et al. (2019) found only a very small relationship between ability and intuitive reasoning as measured by the effect of conflict in belief judgments in 1 out of 3 experiments. 


\section{Experiment 3A}

The results of the first two experiments showed that pseudo-logical validity interferes with belief judgments in the same way that logical validity does. These findings indicate that the so-called logical intuition may not be really about formal logical structure, but about some other problem features that just happen to align with logical validity. For example, it is quite possible that reasoners were exploiting a superficial content matching heuristic that could differentiate between both logically valid and invalid arguments as well as their pseudo-valid counterparts. To illustrate, consider the following pseudological (affirmation of the consequent) arguments:

\section{AC-Pseudo-valid:}

If a child is laughing, then it is sad

A child is sad

The child is laughing

\section{AC-Pseudo-invalid:}

If a child is laughing, then it is sad

A child is sad

The child is crying

In order to avoid the complexity of negations in conditional reasoning, we converted all the explicit negations (e.g., laughing vs. not laughing) to implicit ones (e.g., laughing vs. crying). Consequently, all of the pseudo-invalid arguments had conclusions with a new term (e.g. 'crying) that was not mentioned in either premise, whereas the pseudo-valid arguments have conclusions that match a term in the first premise (e.g, laughing). Thus, participants may have rejected the (pseudo-) valid conclusions because the topic of the conclusion seemed irrelevant to the premises. That is, they may have been responding based on a content matching heuristic: endorsing conclusions that contain a term already mentioned in their first premises (i.e., laughing) and rejecting conclusions contain a term not mentioned in their first premises (i.e., crying). This strategy would also be effective for modus ponens arguments where a content matching heuristic would deliver logically accurate responses. The rapid application of the strategy may also result in the interference observed under belief instructions. 
In order to test whether participants used a content matching heuristic in their intuitive judgments, we added modus tollens (MT) and denial of the antecedent (DA) conditional arguments in Experiment 3A. MT arguments were determinately valid and invalid, whilst all DA arguments were pseudo-valid and pseudo-invalid. As you can see below, in contrast to our earlier expreiments, this time, the (pseudo) valid arguments DA have conclusions with a new term not mentioned in their first premise:

\section{DA - Pseudo-valid:}

If a child is laughing, then it is sad

A child is crying

The child is happy

\section{DA - Pseudo-invalid:}

If a child is laughing, then it is $\underline{\mathrm{sad}}$

A child is crying

The child is sad

Thus, if participants are relying on a content matching heuristic, then this heuristic would produce systematically wrong answers, given that reasoners should reject the (pseudo-) valid forms and accept the (pseudo-) invalid ones, leading to low rates of accuracy on both MT and DA arguments. In addition, MT and DA arguments are more complex than MP and AC arguments, so that Experiment 3A also allows us to investigate the impact of argument complexity on (pseudo-) logical intuition effects as well.

\subsection{Method}

\subsubsection{Participants}

One hundred and twenty-two students from Macquarie University participated in this experiment ( 85 were female, $M$ age $=22.85, S D=8.04$ ). In exchange for their participation, students received two course credits.

\subsubsection{Materials}

In this experiment, we added modus tollens (MT) and denial of the antecedent (DA) conditionals to the task. We reduced the number of inferences of each type to ensure that the total number of 
inferences was the same. Thus, we used 16 arguments of each problem type. Each argument type consisted of 8 conflict and 8 non-conflict items. We created conflict items by crossing logic and believability for MP and MT arguments and by crossing pseudo-logic and believability for AC and DA arguments. In our analyses, we considered MP and MT arguments as "Logical Arguments" and AC and DA arguments as "Pseudo-Logical Arguments". An example of each argument type can be found in Table 1. Moreover, consistent with previous studies (e.g., Ricco et al., 2020; Schaeken \& Schroyens, 2000; Schroyens et al., 2000, 2001; Schroyens \& Braem, 2011; Trippas et al., 2017), we labelled MP and $\mathrm{AC}$ as simple arguments and MT and DA as more complex arguments. We used the same individual differences measures used in Experiment 2.

\subsubsection{Procedure}

Participants first were presented with individual differences measures and then the experimental part of the experiment. Besides adding two more argument types, the procedure of the experiment was the same to Experiment 2.

\subsection{Results}

The average accuracy on the AH4, CRT-3, and CRT-4 was $38.38(S D=10.7), 1.02(\mathrm{SD}=1.17)$, and $1.56(\mathrm{SD}=1.31)$, respectively. The accuracy in these measures were similar to previous studies with student samples (e.g., Frederick, 2005; Ghasemi, Handley, \& Howarth, 2021; Toplak et al., 2014; Trippas et al., 2013) and also to Experiment 2. There were moderate correlations between these three measures $(r s>.34, p s<.001)$. As in Experiment 2, A single measure of cognitive ability was created by combining AH4, 3-item CRT, and 4-item CRT ( $\alpha=.75)$. We performed a median split on cognitive ability scores and created a low ability group $(n=61)$ and a high ability group $(n=61)$.

We performed a GLMM on the accuracy data with conflict, argument type, instruction, complexity, and cognitive ability as fixed factors. The model also contained by-participant random intercept, byparticipant random slope for conflict and instruction, and their correlation, by-item random intercept, by-item random slope for conflict, instruction, cognitive ability, complexity. The results of this model, as is shown in Figure 3, revealed main effects of conflict, $\chi^{2}(1)=35.13, p<.001$, argument type, $\chi^{2}(1)$ 
$=6.63, p=.01$, and general ability, $\chi^{2}(1)=17.69, p<.001$. Participants were more accurate on nonconflict than conflict arguments (.94 vs. .76), and on logical than pseudo-logical arguments (.88 vs. .86). Higher ability participants were more accurate than lower ability participants (.92 vs. .82).

\subsubsection{Pseudo-Intuitive Logic Effect}

The main goals of Experiment 3A were to replicate the pseudo-logic effect that we found in Experiments 1 and 2, and to investigate if this effect interacts with cognitive ability and argument complexity. The results revealed a conflict by instruction interaction, $\chi^{2}(1)=11.34, p<.001$. The effect of conflict, whilst significant for both instructions $(p<.001)$, was more marked under logic instructions (.22 vs. .14). Consistent with Experiments 1 and 2, the effect of conflict under belief instructions, as the main index of logical intuition, was present for both logical and pseudo-logical arguments, as the threeway interaction of conflict, instruction, and argument type was non-significant, $\chi^{2}(1)=.05, p=.82$. This symmetrical pattern can be seen in Figure 3, where the pattern for pseudo-logical arguments mirrors the pattern of logical arguments.

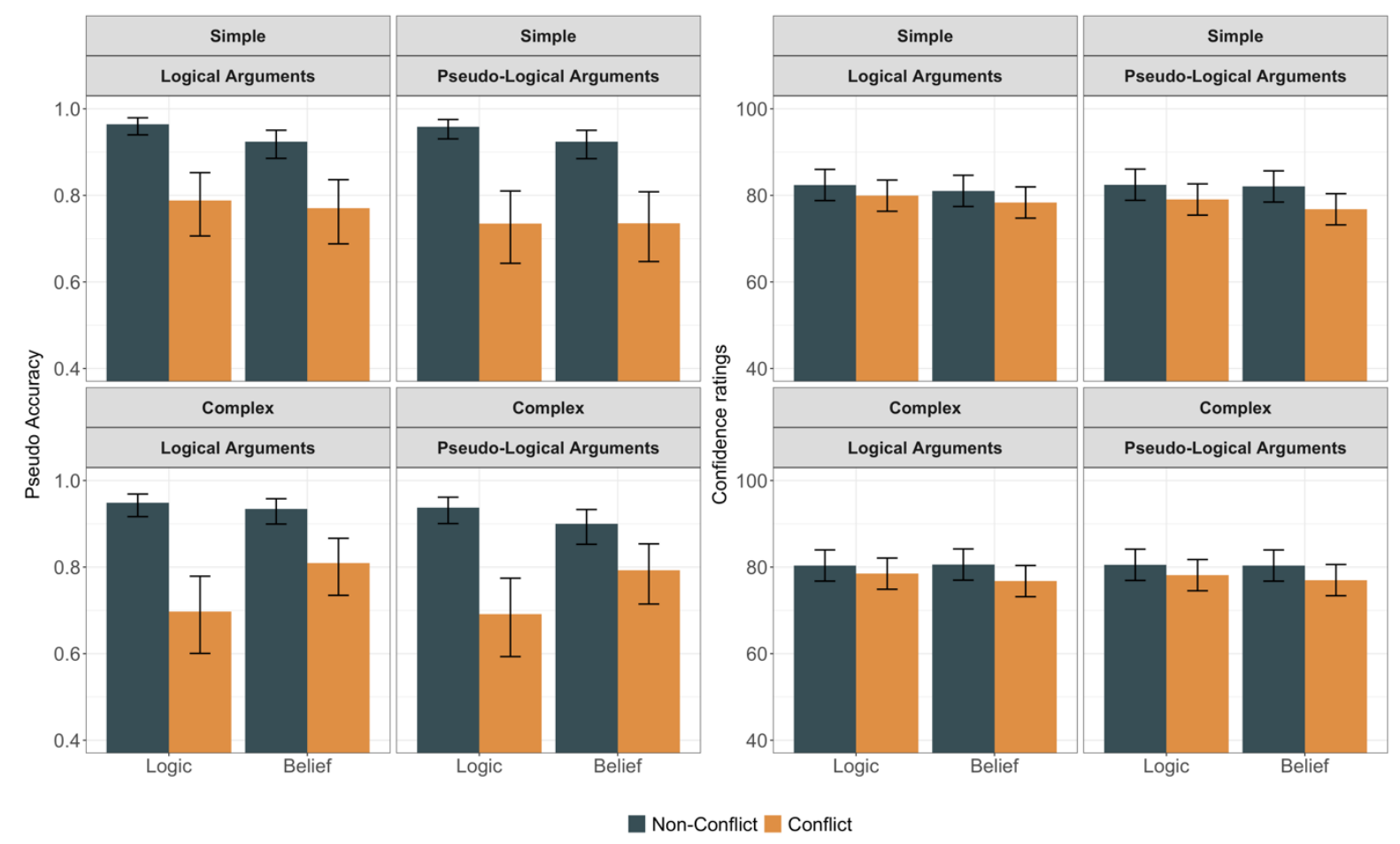

Figure 3: Estimated mean accuracy and confidence ratings for conflict and instruction conditions across argument type and complexity conditions of Experiment 3 A. Error bars represent $95 \%$ confidence interval. 


\subsubsection{The Effect of Complexity}

Moreover, the nonsignificant interaction of conflict, instruction, and argument type was not qualified by a higher order interaction with complexity, $\chi^{2}(1)=.17, p=.67$. In other words, logic interfered with belief judgments to the same extent for high and low ability participants and also for simpler and more complex arguments. The interference of (pseudo-) logic with belief judgments on both complexity levels indicates that a superficial content matching heuristic is unlikely to be the mechanism underlying the "pseudo-intuitive logic" effect. Finally, we found an instruction by complexity interaction, $\chi^{2}(1)=11.34, p<.001$. Post-hoc analysis showed a significant higher accuracy on logic instructions for simple arguments than complex ones $(p=.002)$.

\subsubsection{The Effect of Cognitive Ability}

The main three-way interaction of conflict, instruction, and argument type was not qualified by a higher order interaction with cognitive ability, $\chi^{2}(1)=.29, p=.59$, which indicates that the interference of logic with belief on logical and pseudo-logical arguments was similar for both cognitive ability groups. Moreover, a cross-over instruction by cognitive ability interaction, $\chi^{2}(1)=7.74, p=.005$, indicated that high ability participants had higher accuracy on logic instructions than low ability participants do $(p=.03)$, whilst low ability participants were more accurate, although non-significantly ( $p=.23$ ), on belief instructions. This finding is in line with the general dual-process theory predictions (Evans \& Stanovich, 2013).

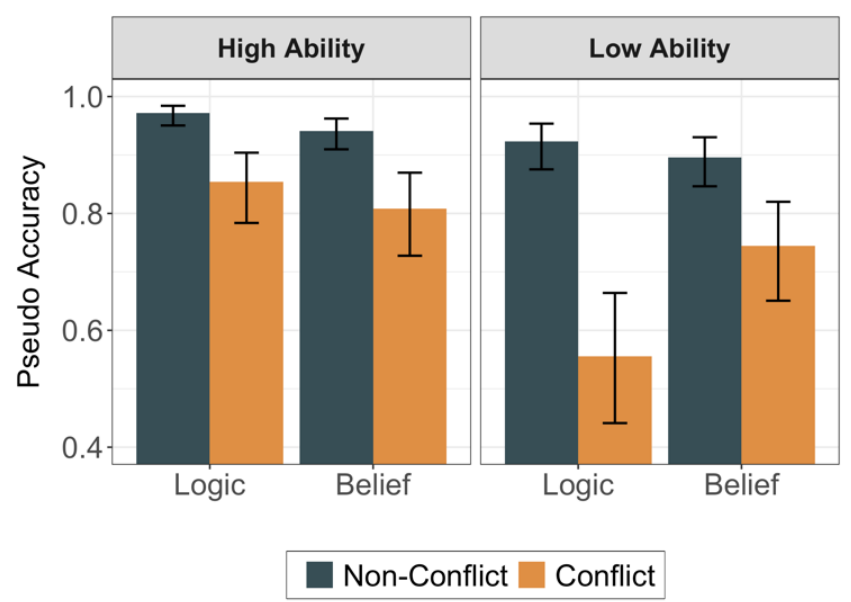

Figure 4: Estimated mean accuracy for conflict and instruction conditions across cognitive ability groups of Experiment 3 A. Error bars represent $95 \%$ confidence interval. 
Finally, as Figure 4 shows, we found a three-way interaction of conflict, instruction, and cognitive ability, $\chi^{2}(1)=5.94, p=.01$. Similar to Thompson et al. (2018), for low ability participants, conflict interfered more with logic judgments than belief judgments, whilst for higher ability participants, conflict interfered with both judgments to the same extent. In other words, the difference between nonconflict and conflict accuracy under belief instructions was similar for both groups (.13 vs. .15 for high and low ability groups, respectively; $\mathrm{p}=.68$ ). However, this difference under logic instructions was more pronounced for the low ability group (.12 vs. .37 for high and low ability groups, respectively; $p$ $<.001)$.

\subsubsection{Confidence Ratings}

A linear mixed model was performed on confidence ratings data. This model included conflict, instruction, argument type, complexity, and cognitive ability as fixed-effect factors, by-participant random intercept and slope for conflict, instruction, argument type, and complexity, by-item random intercept, and by-item random slope for instruction, cognitive ability, and complexity. Consistent with Experiments 1 and 2, we found a marginally significant interaction of conflict and instruction, $F(1$, $7136.92)=3.73, p=.05$, indicating a greater effect of conflict under belief instructions than logic instructions (3.79 vs. 2.54). Most importantly, the three-way interaction of conflict by instruction by argument type was not significant, $F(1,7141.88)=0.07, p=.80$.

\section{Experiment 3B}

In Experiment 3A, in order to test whether the (pseudo-) intuitive logic effect arose due to a simple content matching heuristic, we used implicit negations which meant that such a strategy, if employed, would not work for denial inferences. However, as one of the reviewers pointed out, using implicit negation could make some of our valid MT arguments logically invalid. For example, consider the modus tollens argument that "If a child is laughing, then it is happy; A child is sad; The child is crying". We considered such an argument as a true valid argument in the previous experiment, however, strictly speaking, the conclusion does not necessarily follow from premises as "laughing" and "crying" are not opposites of each other, and therefore don't necessarily negate each other. Whilst we think it is unlikely, 
it is possible that some participants may have interpreted the arguments in this way. For implicit negations to produce valid arguments on MT problems, the conclusion needs to clearly deny the antecedent clause. This is most readily achieved through employing opposites, such as "wet/dry" and "in/out of the water" (see example in Table 1). We did indeed inadvertently include a small number of items where the conclusion to the MT argument may not have been interpreted as clearly denying the antecedent clause. Whilst there were a small number of these items overall $(8 \%$ of all trails in Experiment 3), more than half of the valid MT trials were affected in this way. Although the hierarchical nature of our analysis would take into account idiosyncrasies in items, we replicated the analyses in Experiment 3A after excluding all valid MT arguments in which the conclusion did not deny the antecedent clause. Two random intercept models for pseudo-accuracy and confidence data replicated the key findings. The results of these analyses can be found in the online materials (See Tables S14 and S15).

Although these findings suggest that our results were not affected by potential variation in argument interpretations arising from our use of implicit negation, we decided to replicate Experiment 3A with arguments consisting of explicit negation. For example, the modus tollens arguments above was changed to "If a child is laughing, then it is happy; A child is not happy; The child is not laughing". As the result of applying explicit negations to construct the arguments, all conclusions contain a term already mentioned in the first premises. Thus, the results of this experiment cannot be used to test the content matching heuristics. Since the main goal of this experiment is to replicate the key findings of Experiment $3 \mathrm{~A}$ regarding the effect of complexity, we excluded individual differences measures. Unless explicitly mentioned, all other characteristics of the two experiments were the same.

\subsection{Method}

\subsubsection{Participants}

For this experiment, we recruited 132 undergraduate students, including 91 students from Macquarie University and 41 students from the University of Saskatchewan ( 86 were female, $M$ age $=$ $20.08, S D=4.05)$. Participants received one course credit to take part in the experiment. 


\subsubsection{Materials}

We modified all arguments of Experiment $3 \mathrm{~A}$ by converting implicit negations to explicit ones. In order to avoid double negation, we replaced three arguments of Experiment $3 \mathrm{~A}$ with more suitable arguments from Experiments 1 and 2. Thus, we ended up with 64 arguments in total, and 16 arguments for each problem type.

\subsubsection{Procedure}

Except for the exclusion of individual differences measures, the same procedure as Experiment 3A was used.

\subsection{Results}

A GLMM with conflict, argument type, instruction, and complexity as fixed factors was performed on the accuracy data. This model included by-participant random intercept and slope for conflict, instruction and complexity, a correlation term for by-participants random effects, and by-item random intercept and slope for instruction and complexity. As is shown in Figure 5, the results of this model revealed main effects of conflict, $\chi^{2}(1)=102.35, p<.001$, complexity, $\chi^{2}(1)=10.27, p<.001$, and instruction, $\chi^{2}(1)=9.5, p=.002$. Participants were more accurate on non-conflict than conflict arguments (.95 vs. .76), on simple than complex arguments (.90 vs. .87), and under logic than belief instructions (.92 vs. .86).

\subsubsection{Pseudo-Intuitive Logic Effect}

Consistent with Experiment $3 \mathrm{~A}$, the results revealed a conflict by instruction interaction, $\chi^{2}(1)=$ $17.72, p<.001$. The effect of conflict, whilst larger under logic instructions than belief instructions on a $\log$ odd scale ( 8.33 vs. 4.46 ), was not different under both instructions on the response scale (.18 vs. .19). More importantly, consistent with Experiments 1, 2, and 3A, the conflict interfered with belief judgments on both logical and pseudo-logical arguments, as the three-way interaction of conflict, instruction, and argument type was non-significant, $\chi^{2}(1)=.01, p=.91$. Thus, the conflict effects were observed with both the implicit and explicit negations. 


\subsubsection{The Effect of Complexity}

First, a visual comparison of Figures 3 and 5 suggests a very similar pattern of responding in terms of accuracy and confidence ratings in both experiments. As in Experiment 3A, the conflict by instruction by argument type interaction was not qualified by a higher order interaction with complexity, $\chi^{2}(1)=.33, p=.57$. In other words, logic interfered with belief judgments on both simpler and more complex arguments. Figure 5 shows reliable conflict effects under belief instructions. Such an effect, however, was smaller for Complex pseudo-logical arguments. The results revealed that instruction interacted with complexity, $\chi^{2}(1)=11.58, p<.001$, which indicates a significant effect of argument type only under logic instructions $(p<.001)$, but not belief instructions $(p=.48)$. Moreover, we found a conflict by argument type by complexity interaction, $\chi^{2}(1)=22.01, p<.001$, which indicates that, on simple arguments, conflict interfered more with pseudo-logical arguments than logical ones, and on complex arguments, there was a larger conflict effect on logical arguments than pseudo-logical ones.

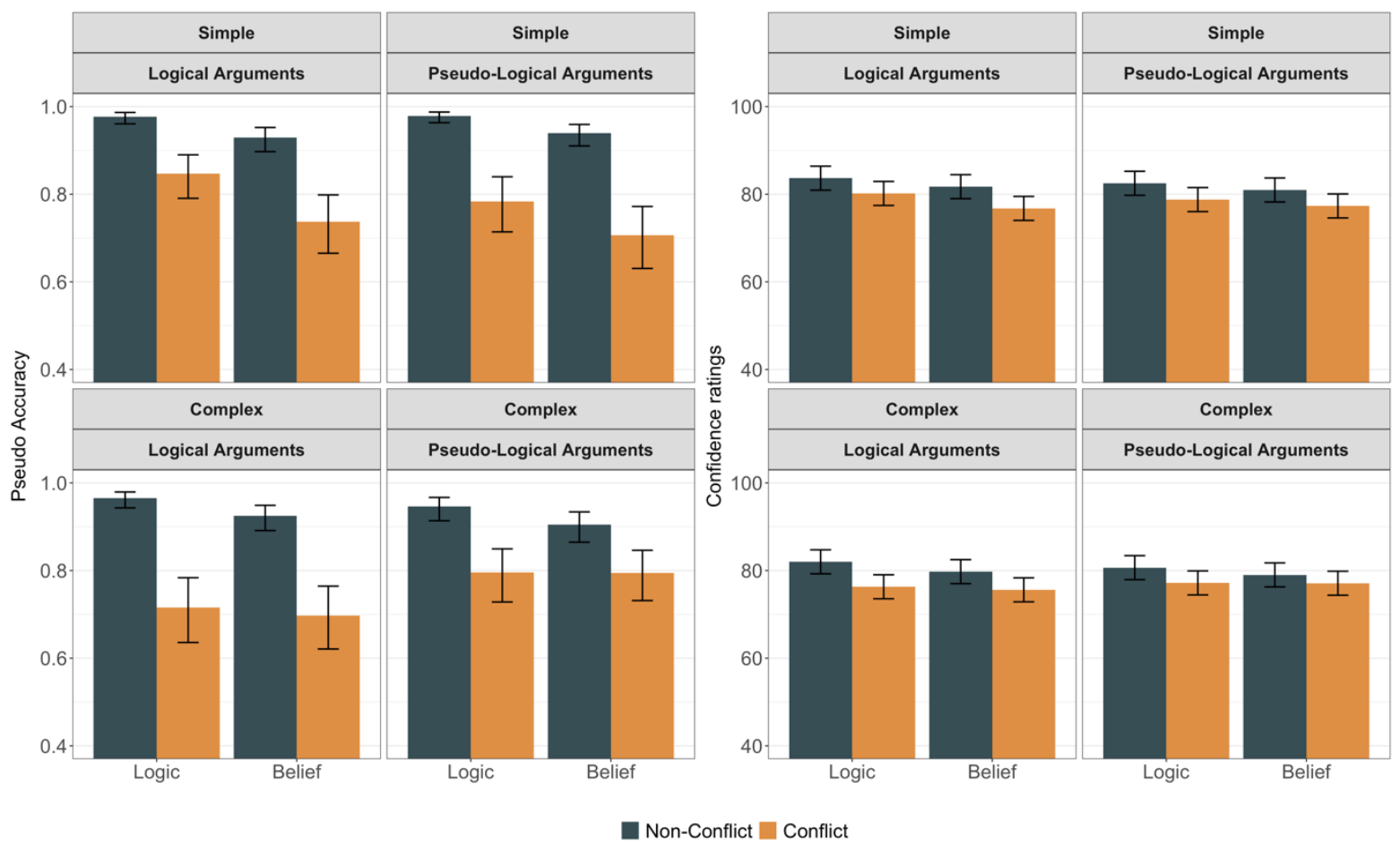

Figure 5: Estimated mean accuracy and confidence ratings for conflict and instruction conditions across argument type and complexity conditions of Experiment 3B. Error bars represent $95 \%$ confidence intervals. 


\subsubsection{Confidence Ratings}

A linear mixed model with conflict, instruction, argument type, and complexity as fixed-effect factors, by-participant random intercept and slope for conflict, instruction, and complexity, and by-item random intercept was performed on confidence ratings data. Consistent with the first 3 experiments, the three-way interaction of conflict by instruction by argument type was not significant, $F(1,7906.40)=$ $.36, p=.55$. This effect was not qualified by a higher order interaction with complexity, $F(1,7903.03)$ $=.33, p=.57$.

\subsubsection{Conflict Detection}

Thus far the findings across all three experiments suggest that conflict impacts on belief judgments irrespective of whether the conflict is based upon logical validity or conclusion plausibility. The effect of belief/logic conflict on belief judgments may indicate the rapid, default activation of an inference that then interferes with the evaluation of conclusion believability. Another well-known method to study logical intuition, is the conflict detection paradigm, which has shown that reasoners have lower confidence and higher response latency for conflict arguments compared to non-conflict arguments (Bago \& De Neys, 2017; De Neys \& Glumicic, 2008; Frey et al., 2017; Thompson \& Johnson, 2014). Importantly, these studies have shown that reduced confidence and increased latency are present even when participants give an incorrect response. This shows that logic seems to affect responding even when the participants have given the non-logical response.

In this section, we report a similar analysis as an additional means of testing whether both logical and pseudo-logical inferences are available intuitively. For this analysis, we pooled all data from three experiments and performed multiple random intercept mixed models on confidence ratings and response latency data. For correct conflict responses, reasoners may have solved the conflict since they have produced the correct response. Moreover, incorrect non-conflict responses may indicate inattentiveness or random responding. Thus, to make the results comparable to other conflict detection studies (Frey et al., 2017; Šrol \& De Neys, 2020), we performed our analyses on correct non-conflict and incorrect conflict responses under logic and belief instructions separately. 
We started with confidence ratings under logic instructions. As figure 6 shows, participants reported higher confidence on non-conflict than conflict arguments, $F(1,9416.53)=220.91, p<.001$, and logical arguments than pseudo-logical ones, $F(1,9225.19)=4.27, p=.04$. Crucially, conflict and argument type did not interact $(F=.32, p=.57)$, which indicated that the effect of conflict on pseudological arguments mirrored that of logical arguments. For belief instructions, our model revealed higher confidence on non-conflict arguments than conflict ones, $F(1,9181.73)=640.22, p<.001$. No other main effects and interactions were significant $(F s<.5, p s>.48)$. Thus, in line with previous findings, the conflict interfered with both logic and belief judgments to the same extent for logical and pseudological arguments.
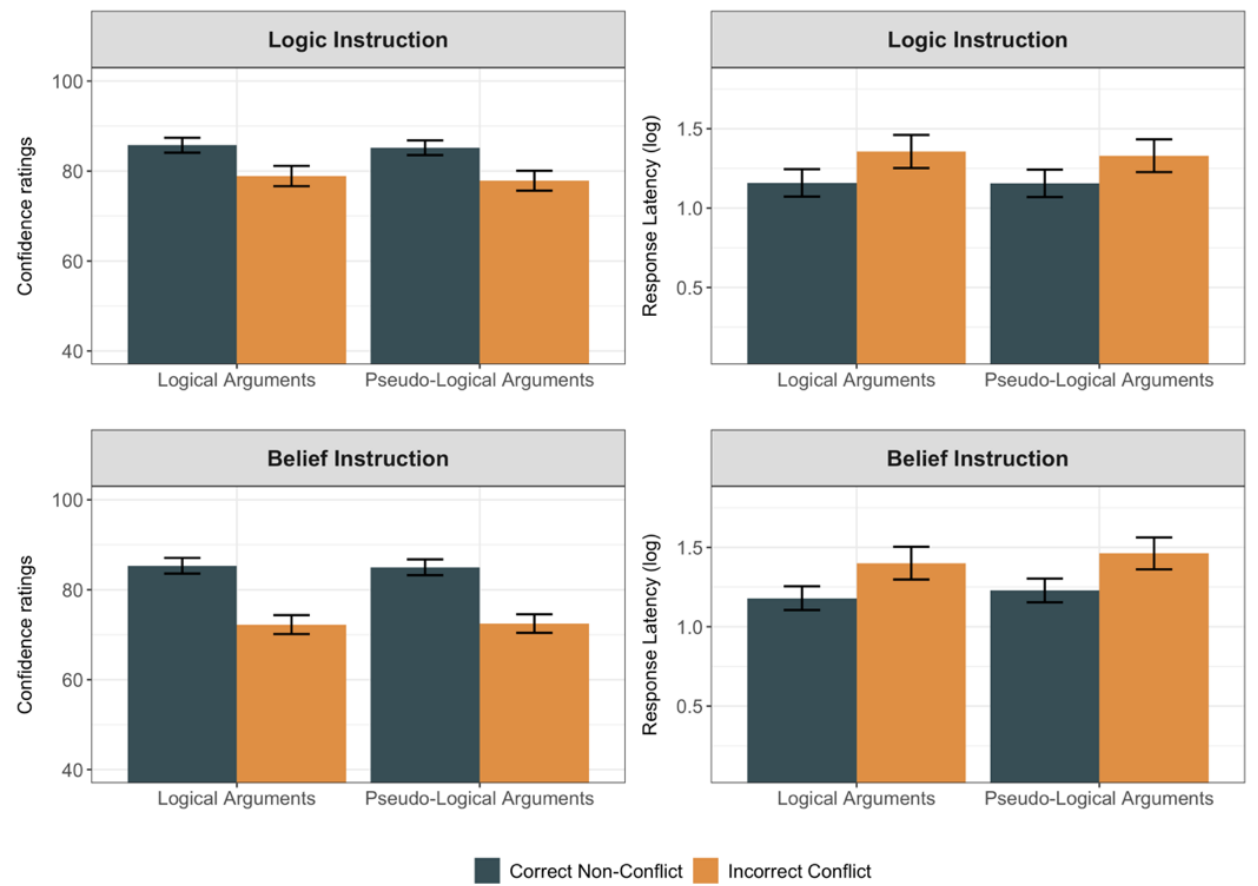

Figure 6: Confidence ratings and response latencies for incorrect conflict and correct non-conflict problems across logical and pseudo-logical arguments.

Next, we performed two random intercept mixed models on log-transformed response latency data. Since no reaction time was recorded on Experiment 1, we only included data from the second two experiments. The model for logic instructions revealed a main effect of conflict, $F(1,7372.03)=69.09$, $p<.001$, which indicated that participants had higher latency in responding to conflict arguments compared to non-conflict arguments. The main effect of argument type and the two-way interaction 
were not significant $(F s<1.05, p s>.31)$. For belief instructions, we found higher response latencies in responding to conflict, $F(1,7252.44)=69.49, p<.001$, and pseudo-logical argument, $F(1,7027.08)$ $=5.83, p=.02$. This finding is in line with previous findings of the current study regarding the effect of conflict on accuracy and confidence ratings under belief instructions. More importantly, these two factors did not interact $(F=.73, p=.39)$. Both analyses of confidence ratings and response latencies suggested that conflict detection did not differ for pseudo-valid and valid arguments, which is further evidence that logical intuitions are not necessarily based on logical validity.

\subsubsection{A Test of the Null Hypothesis}

The goal of this experimental series was to test the logical intuition account, according to which, underlying logical structures conflict with belief judgments. In contrast with such an account, we found that both actual logical structures and pseudo-logical structures interfere with belief judgments as the three-way interactions of conflict by instruction by argument type are found to be non-significant. However, we have claimed that the "absence of evidence" in favor of a logic-based account provides strong support for a non-logical heuristic explanation of intuitive logic. As non-significant effects cannot be interpreted as the absence of an effect in the frequentist approach (Aczel et al., 2018), in this section, using Bayesian parameter estimation and model comparison approach (Kruschke, 2014; Kurz, 2021), we offer the "evidence of absence" by showing that the conflict effect was not significantly larger on logical arguments than pseudo-logical ones.

For each experiment, we ran a hierarchical Bayesian mixed model using the brms package (Bürkner, 2017). Each model contained conflict, instruction, and argument type and their interactions as fixed factors and a maximal random effect structure. The exact structures of these models, their prior specification, likelihood distributions, and convergence and posterior predictive checks can be found in the online materials. Figure 7 summarises posterior predictive distributions of the conflict effect (i.e., non-conflict - conflict) under belief instructions for both logical and pseudo-logical arguments. This figure shows that conflict effects have similar magnitudes on logical and pseudo-logical arguments as the $95 \%$ credible intervals of the contrast distributions (logical - pseudo-logical panel) include zero. In 
Experiment 2, the credible interval barely excludes zero, but as discussed in the result sections of that experiment, it indicates a larger conflict effect on pseudo-logical arguments than logical ones, which is in contrast with the true intuitive logic hypothesis.

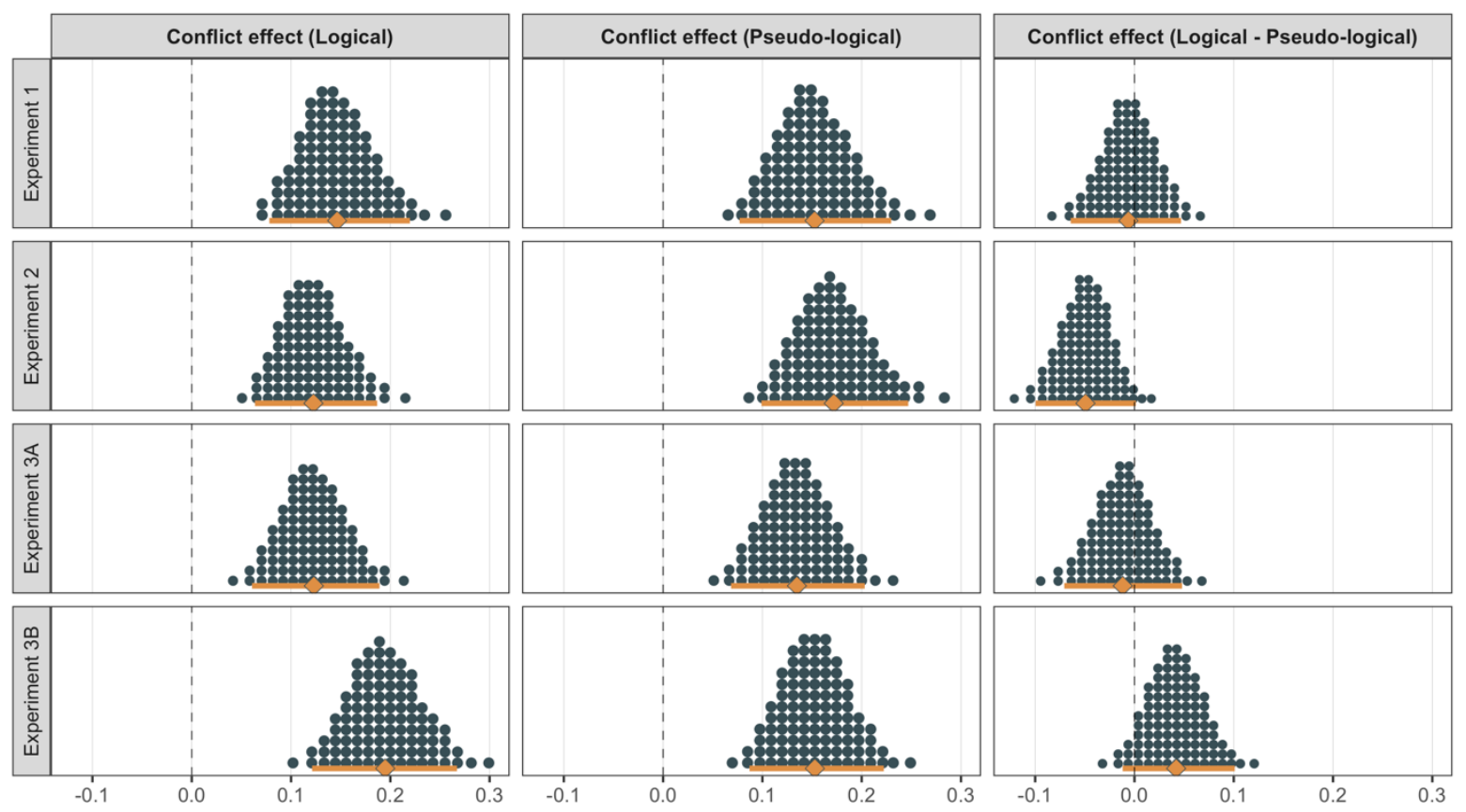

Figure 7: Posterior predictive distributions of conflict effects under belief instructions for logical and pseudo-logical arguments across all experiments. The central diamond in each distribution represents the posterior mean. We used the highest density interval (HDI) as the 95\% CI.

Next, we employed two model comparison approaches to compare the predictions of our models. First, Bayes factor indexes demonstrated that the data are substantially more likely to occur under the hypothesis that the conflict effect is not different for logical and pseudo-logical arguments, $\mathrm{BF}_{01}=$ 36.45, 1.94, 42.83, 51.88 for Experiments 1, 2, 3A, and 3B, respectively. Finally, using leave-one-out (LOO) cross-validation criteria (Vehtari et al., 2017), we compared each model with a similar model without the 3-way interaction. The results showed that for Experiments 1,3A, and 3B, the models without the 3-way interaction terms were favored over the model with such an interaction term, elpd diff $=-1,-1.1,-1.6$, respectively. For Experiment 2, we found that the model with the interaction term showed to be the best fitting model, elpd diff $=-2.5$, but as explained previously, this interaction indicates a larger conflict effect on pseudo-logical arguments. This also explains the small Bayes factor for the effect of this experiment. Altogether, the results of our Bayesian analyses showed that the conflict effect 
under belief instructions, as the index of intuitive logic, is not different on logical and pseudo-logical arguments, and if anything, it is larger on pseudo-logical ones.

\subsection{Discussion}

Consistent with the first two experiments, in Experiments 3A and 3B, we found that the response pattern for the pseudo-logical arguments mirrored that for the logically valid arguments: Belief judgments were equally impacted by logical and pseudo-logical arguments. These data suggest that, in contrast to the label "logical intuition", participants relied on problem features other than logical validity in their intuitive reasoning. One of the goals of Experiment $3 \mathrm{~A}$ was to test whether a simple content matching strategy could explain the effect of conflict between (pseudo-) logic and belief on belief judgments. The content matching account predicted the conflict effect only for MP and AC arguments but not for MT and DA arguments. However, both the accuracy and confidence analyses showed that (pseudo-) logic interfered with belief judgments on both complexity levels. Thus, a superficial content matching heuristic is unlikely to be the mechanism underlying the "intuitive logic" effect.

In Experiment 2, we observed that low and high ability reasoners were equally sensitive to conflict in the belief and logic instructions conditions. This finding is in contrast to some others suggesting that high ability reasoners might have better developed logical intuitions than their low ability counter-parts (Raoelison et al., 2020; Thompson et al., 2018). We suggested that the explanation for these findings might lie in the simple nature of the inferences used in that experiment. In Experiment $3 \mathrm{~A}$, we used slightly more complex arguments compared to the previous experiment and found that this changed the nature of the conflict effect for low and high ability reasoners: Lower ability participants showed more of an effect of beliefs on logic judgments compared to high ability participants. This finding is largely congruent with the conclusion that higher ability participants are less impacted by belief in their logic judgments and hence are more accurate in their logical reasoning (Thompson et al., 2018; Toplak et al., 2011, 2014). However, belief interfered with logic judgments to the same extent for both cognitive ability groups. Consistent with Experiment 2, this finding supports the notion that individual differences 
in logical intuition cannot be explained by variation in cognitive ability (Ghasemi, Handley, \& Howarth, 2021; Howarth et al., 2018; Markovits et al., 2020; Morsanyi \& Handley, 2012).

The results of the conflict detection analysis indicated that the conflict effect (i.e., lower confidence and higher response latency on conflict problems compared to non-conflict ones), which has been considered as one of strongest evidence of intuitive logic, was present for both logical and pseudological arguments. Similarly, Bayesian parameter estimation and model comparison approaches revealed the same findings by providing evidence in favor of a non-logical heuristic hypothesis of intuitive logic. In other words, the conflict between logic and belief affected participants' responses in the same way that the conflict between pseudo-logic and belief did. These findings are inconsistent with the claim that conflict effects are driven by considerations of logical validity.

\section{General Discussion}

In three experiments, we tested the hypothesis that reasoners have intuitive access to logical validity. This research was motivated by findings that logical validity interferes with the ability to process conclusion believability (Handley et al., 2011), along with the assumption that the mechanism underlying this phenomenon can be described as "intuitive logic" (De Neys \& Pennycook, 2019; Handley \& Trippas, 2015; Thompson \& Newman, 2017). That is, it has been assumed that reasoners are able to process the logical structure of an argument so quickly and intuitively that it interferes with their ability to process conclusion believability (Pennycook et al., 2014; Thompson et al., 2018; Trippas et al., 2017). Our data strongly challenge this interpretation, in that we have demonstrated that invalid arguments also interfere with reasoners' ability to judge conclusion believability. Thus, it seems unlikely that the mechanism that gives rise to "intuitive logic" is based on logical validity per se; instead, reasoners are likely processing some other regularity in the stimulus materials that produces conflict with belief judgments. Below, we discuss several possible candidates for the underlying regularity. 


\subsection{Candidate explanations for the source of Logical Intuitions}

One argument that could be made for a logical structure underlying the conflict effect is a biconditional reading of the conditional premise. Perhaps reasoners interpreted the conditional as a biconditional and were therefore showing an intuitive logic effect, albeit one that relied on a particular logical interepretation of the first premise? Whilst this is possible, it is inconsistent with the large body of evidence showing that acceptance of the $\mathrm{AC}$ inference arises for pragmatic reasons having to do with the availability of alternative antecedents (Markovits \& Potvin, 2001; Rumain et al., 1983; Thompson, 1994; see also Evans \& Over, 2004 for additional arguments against a biconditional reading). Further evidence that accepting $\mathrm{AC}$ is due to pragmatic processes is that reasoners draw $\mathrm{AC}$ less often when they receive 'strict logical instructions' that emphasise the importance of only drawing conclusions that necessarily follow from the premises (Evans et al., 2010; Schroyens et al., 2003). Similarly, when reasoners are given unlimited time to respond, AC rates tend to decrease (Newman et al., 2017; Schroyens et al., 2003) and the rejection of AC is associated with longer reaction times than endorsement (Bonnefond et al., 2012). Interestingly, although AC inferences are drawn readily in text comprehension, there is no evidence that this arises from a biconditional interpretation (Rader \& Sloutsky, 2002). It has been shown that reasoners of higher ability, compared to low ability reasoners, tend to reject the $\mathrm{AC}$ inference more readily, but endorse MP inferences at higher rates (Evans et al., 2010; Newstead et al., 2004).

Whilst there is reasonably convincing evidence in the literature against a routine biconditional interpretation of conditional assertions, is there any evidence that our participants were less likely to treat $\mathrm{AC}$ arguments as valid compared to MP? To test this, we performed an analysis on aggregated data with (pseudo-) validity (Valid vs. Invalid) and argument type (MP vs. AC) on endorsement rates under logic instructions. The results revealed a significant validity by argument type interaction, $F(1$, $11602.41)=8.40, p=.004$, indicating that the (pseudo-) validity effect, whilst significant for both argument types $(p s<.001)$ was larger for MP arguments (.65 vs. .61). Thus, we have some limited evidence that sensitivity to (pseudo) validity is greater for MP than AC under logic instructions which is consistent with the idea that biconditionality does not underpin the intuitive logic effects. This 
analysis, coupled with the evidence based arguments described in the previous paragraph, support the view that AC, whilst readily drawn by many reasoners (e.g., Evans et al., 1995), is not inferred based upon a fixed representation that corresponds to a biconditional interpretation.

An alternative representational explanation is based upon the mental model theory of reasoning (Johnson-Laird \& Byrne, 1991, 2002), which states that reasoners form an initial representation of the conditional, which, if not fleshed out further, gives rise to the $\mathrm{AC}$ inference. According to the mental model theory, reasoners construct an initial superficial representation of conditional premises that supports AC, as illustrated below. This model represents the single possibility in which both antecedent and consequent are true:

[A] 3

The elipsis '...' is a placeholder indicating that there are other possibilities consistent with the conditional that are not yet explicitly represented. When reasoners are given the AC minor premise, ' 3 ', they will often infer that ' $\mathrm{A}$ ' holds because the initial model supports this inference. However, it can be rejected if the initial model is 'fleshed out' into the full set of three possibilities, because the second model contradicts the inference:

A $\quad 3$

Not-A 3

Not-A Not-3

In this view, the $\mathrm{AC}$ inference is plausible because it is based upon an initial and superficial representation of the conditional premise and it is hence likely to be readily available. The results of Experiments 1 and 2 are consistent with the initial representation account, according to which, a similar pattern of findings on modus ponens (MP) and affirmation of the consequent (AC) arguments is expected since both inferences are derived from the same initial representation of the premise. The initial representation hypothesis, however, cannot explain the findings of Experiments $3 \mathrm{~A}$ and $3 \mathrm{~B}$, where interference effects were observed for DA and MT inferences, which are not supported by the 
initial representation (Schroyens \& Braem, 2011). Thus, the fact that participants showed sensitivity to (pseudo-) logic on both simple and complex arguments in Experiments 3A and 3B makes the initial representation account a less plausible candidate to explain the (pseudo-) intuitive logic effect.

The results of Experiment 3A also ruled out a simple content matching heuristic as another possible mechanism of (pseudo-) intuitive logic. As outlined in the introduction to Experiment 3A, it is possible that participants endorse valid MP and AC arguments simply because the valid inferences have the same terms in their premises and conclusions whilst the invalid inferences had conclusions with a new term that was not mentioned in either premise. In Experiment 3A, we tested this account directly by using MT and DA arguments in which the valid forms, not the invalid ones, had conclusions with the new term that was not mentioned in the conditional premise. If a content matching heuristic underlies reasoners' judgment, then participants should have rejected the (pseudo-) valid forms and endorsed the (pseudo-) invalid ones for the more complex arguments. Contrary to this prediction, the results revealed that participants still endorsed (pseudo-) valid inferences and rejected (pseudo-) invalid inferences.

However, the idea that reasoners might employ a heuristic strategy that draws upon the structural features of an argument is a realistic possibility. Thus, we consider that a simple syntactic matching heuristic might produce the conflict effects observed in our three studies. This heuristic is similar to the atmosphere heuristic that has been identified in syllogistic reasoning (Wetherick \& Gilhooly, 1995), which suggests that positive premises should lead to positve conclusions and negated premises lead to negated conclusions. As can been seen in Table 1, in valid MP (If $p$ then $q$; $p$; therefore, $q$ ) and pseudovalid AC (If $p$ then $q$; $q$; therefore, $p$ ) arguments, the positive $p$ and $q$ elements of the conditionals go together. The invalid version of MP and the pseudo-invalid version of AC both violate this pattern, pairing a positive proposition with a negated conclusion: (If $p$ then $q$; $p$; therefore, not- $q$ ) and (If $p$ then q; q; therefore, not-p), respectively. A matching heuristic can also explain performance on the MT and DA forms. The valid versions of these arguments pair two negations (If $p$ then $q$; not- $q$; therefore, not$p$ ) and (If $p$ then $q$; not-p; therefore, not- $q$ ) respectively, but the invalid version pair a negated proposition with a positive conclusion: (If $p$ then $q$; not- $q$; therefore, $p$ ) and (If $p$ then $q$; not-p; therefore, 
$q$ ). Thus, a syntactic heuristic would account for acceptance of (psuedo) valid forms and rejection of (pseudo-) invalid arguments in all three studies. Although it is speculative, it is a plausible explanation for our findings. However, we note that additional work is required to understand the limits of such a matching strategy. In particular, it will be important to examine the conditions under which it will be activated and how it differs from a deliberative conditional reasoning strategy.

\subsection{Individual Differences in Logical Intuitions}

Our results showed that intuitive logic, as is measured by the effect of conflict on belief judgments, was unrelated to cognitive abilities. This lack of a relationship between individual differences in cognitive ability and accuracy on both logical and pseudo-logic arguments is surprising. These findings are in contrast to several previous studies which have shown that higher ability reasoners have stronger logical intuitions than low ability reasoners. For example, Šrol \& De Neys (2020) found that conflict detection efficiency, an index of logical intuition, is related to cognitive ability and cognitive style. Similarly, using the two-response paradigm, Raoelison et al. (2020) found that the relationship between ability and intuitive accuracy (first response) was stronger than the relationship between ability and deliberative accuracy (second response). Finally, Thompson et al. (2018) observed that for high ability participants, conclusion believability interfered with logic judgements, whereas the reverse was true for low ability participants. These results strongly suggest that individual differences in logical intuitions are positively correlated with cognitive capacities. However, studies that have examined logical intuitions by manipulating instructions (e.g., asking reasoners whether they like conclusions), have not consistently observed that the validity effect on liking judgements was related to cognitive abilities (Ghasemi, Handley, \& Howarth, 2021; Howarth et al., 2018; Nakamura \& Kawaguchi, 2016).

At this point, it is not clear how to reconcile these seemingly contradictory results. There appears to be two variables that might be at play. One is the degree to which reasoners are asked to explicitly respond based on logical validity; on these tasks there seem to reliable individual differences, such that higher ability people have stronger logical intuitions. Our own results seem to be the one exception to that generalization. We posit that the relative simplicity of our materials may have equalled the playing 
field somewhat. Trippas et al. (2017), for example, demonstrated that simple logical rules interfered more strongly with belief judgments than complex logical rules. It is possible that the inferences that we used are so simple that all participants have access to the matching strategy, and that we would have needed to use more complex syllogistic structures to observe a relationship between logical accuracy and cognitive ability. Consistent with this analysis, using more demanding inferences in Experiment 3A, we established a relationship between cognitive abilities and accuracy under logic instructions, but there was no evidence that this extended to accuracy on conflict problems under belief instructions, our principal indicator of intuitive logic. We believe this issue is ripe for further investigation, e.g., to examine individual differences in logical and pseudo-logical inferences which demand more complex strategies than a simple matching heuristic for evaluation.

\subsection{Implications For Intuitive Logic Accounts}

The concept of "logical intuition" was introduced to describe phenomena whereby reasoners appeared to respond intuitively based on normative rules, such as logical validity. As has already been reviewed (see Table S1 in the online materials), logical intuition is a well-established effect across different reasoning tasks, arguments, and paradigms. For example, this effect has been found on baserate tasks (Frey et al., 2017; Pennycook et al., 2015), conditionals reasoning tasks (Newman et al., 2017; Trippas et al., 2016), the bat-and-ball task (Bago et al., 2019; Raoelison et al., 2020), and ratio bias problems (Mevel et al., 2015). Moreover, different research paradigms including conflict detection (Bago \& De Neys, 2020; De Neys, 2012), misattribution (Ghasemi, Handley, \& Howarth, 2021; Trippas et al., 2016), instructional manipulations (Pennycook et al., 2014; Trippas et al., 2017), and tworesponse (Bago \& De Neys, 2019; Thompson \& Johnson, 2014) paradigms have reached the same conclusion regarding the existence of intuitive logic. The current study, however, has demonstrated that reasoners may, instead, be sensitive to non-logical problem features that may or may not align with logical validity. Our results invite researchers to consider whether, for any given paradigm, it is necessary to propose that the "logical intuition" phenomenon can really be best described by the automatic application of a normative rule or by a simpler, heuristic strategy. Thus, a new line of research 
is called for, to replicate and extend our findings across other domains. Several recommendations for future studies have been listed in the next section.

As we have already explained, it is possible that individuals automatize simple logical and statistical rules during years of education and practice (Stanovich, 2018). The recent findings that demonstrate children develop a more advanced intuitive logic capability as they get older support this account (Raoelison et al., 2021). However, the evidence suggests that people are not very good at applying these rules when it is appropriate to do so, at least for people with lower cognitive abilities (Thompson et al., 2018). Now, one may ask why don't people use the learned logical rules instead of using alternative structural cues? First, we need to note that the heuristic strategies that we proposed as the underlying mechanism of intuitive logic are not something that people learn during development. We believe that these heuristics are strategies that people may develop spontaneously when presented with a novel task. These heuristics sometimes align with logical validity and hence result in a correct response (e.g., in the case of MP arguments) and sometimes do not align with logical validity and cause reasoning errors (e.g., in case of AC arguments). So, why do people use these heuristics instead of the learned logical rules? Some people may not learn formal logic and even those who do may not recognize that it is appropriate to apply them. Moreover, in the absence of the a) knowledge or b) ability to apply logical rules, it makes sense that they would turn to some other element of the task environment to exploit. We argue that such strategies are more superficial than many forms of logical reasoning, such as an exhaustive search for counterexamples in conditional reasoning, a Bayesian updating on base-rate problems, or reductio ad absurdum in the case of modus tollens. This is why we labelled such strategies as "superficial".

To summarise, our results indicate that reasoners were equally sensitive to the conflict between pseudo-logic and belief as they were to the conflict between logic and belief. For this reason, we argue that intuitive logic accounts need to consider the possibility that the underlying mechanism of logical intuition may not be a normative rule but rather a more simple heuristic and this finding will need to be 
incorporated in the new formulization of human reasoning (De Neys \& Pennycook, 2019; Handley \& Trippas, 2015; Thompson \& Newman, 2017).

\subsection{Future Directions}

In the current study, we used 4 forms of conditional inference. The data was analyzed both as they are typically analyzed with instructional manipulation tasks but also the conflict detection analysis that is more typical of the logical intuition research. The results demonstrated that logical validity interfered with belief judgments to the same extent as pseudo-logical structures. However, as one of our reviewers has suggested, future studies could usefully test the (pseudo-) intuitive logic effect more directly by crossing logical cues and structural cues. An interference effect in conditions where logical validity and non-logical structural cues were mis-aligned would be strong evidence against the intuitive logic account $^{4}$.

Moreover, as was pointed out by one of our reviewers, the interference effect that we considered as evidence for (pseudo-) intuitive logic might be a by-product of task-switching rather than logical or pseudo-logical structures. In other words, in trials that participants are cued with a response different than the previous trial (i.e., switch trials), compared to trials that cues a same response (i.e., repeat trials), a carry-over activation may interfere with responding and hence, cause the conflict effect. Contrary to this hypothesis, the interference effect has been found in previous studies that have used a between-subject design (Handley et al., 2011; Howarth et al., 2016; Pennycook et al., 2014). However, to rule out task-switching as a potential explanation for the current data, we completed an additional analysis of the belief instruction condition on aggregated data over the three studies which showed a clear effect of conflict on both switch and repeat trials: conflict did not interact with trial types, $\chi^{2}(1)=$ $.38, p=.54$, and $F(1,15387.87)=1.49, p=.22$, for accuracy or confidence data, respectively (see the online materials for a more detailed discussion). This finding supports the (pseudo-) intuitive logic explanation rather than one based upon task switching. However, it would be valuable to replicate the

\footnotetext{
${ }^{4}$ We would like to thank Wim De Neys for this suggestion.
} 
current findings using between-subject or block designs to test the degree to which task switching demands might moderate the effect ${ }^{5}$.

Finally, future studies could replicate and extend our findings by using different reasoning arguments and tasks in which there is evidence of the application of heuristics. We have argued that a simple matching heuristic is the most plausible mechanism of intuitive judgments for conditional arguments. However, it is quite possible that reasoners employ different strategies for other problem types. For example, reasoners may apply the atmosphere or the min-heuristic (Chater \& Oaksford, 1999) for syllogisms while evaluating spatial inferences based on their coherency rather than logical validity. It would be interesting to examine the generalizability of these findings across other tasks that have produced evidence for logical intuition, such as the brightness judgment task, the liking judgment tasks, or the two-response task. In the current study, we focused on logical validity as the normative rule. Future studies could extend the design to other normative rules such as Bayes Theorem or the conjunction rule, by implementing different reasoning problems such as base-rate or conjunction fallacy problems.

\subsection{Conclusion}

Recently, theorists have suggested that reasoners are intuitively sensitive to normative rules such as logical validity (De Neys, 2012; Handley \& Trippas, 2015; Trippas et al., 2017). We tested this "logical intuition" hypothesis in three experiments. Participants were presented with logical and pseudo-logical arguments and asking them to judge those arguments either based on logical validity or believability. The results revealed that logical validity and pseudo-logic equally interfered with belief judgments, a pattern that was held for simple and more complex inferences, as well as for high and low ability participants. Altogether, these findings suggest that the phenomenon that has been labelled "logical intuition" may have little to do with logic per se, and instead, may be driven by the processing of more superficial structural features that happen to align with logical validity.

\footnotetext{
${ }^{5}$ We would like to thank Karl Christoph Klauer for proposing this alternative explanation and the research design.
} 


\section{Open Science practice}

Online materials, data (Ghasemi, Handley, Howarth, et al., 2021), and analysis scripts can be retrieved at the Open Science framework (https://osf.io/mfhsq/).

\section{References}

Aczel, B., Palfi, B., Szollosi, A., Kovacs, M., Szaszi, B., Szecsi, P., Zrubka, M., Gronau, Q. F., van den Bergh, D., \& Wagenmakers, E.-J. (2018). Quantifying support for the null hypothesis in psychology: An empirical investigation. Advances in Methods and Practices in Psychological Science, 1(3), 357-366.

Bago, B., \& De Neys, W. (2017). Fast logic?: Examining the time course assumption of dual process theory. Cognition. https://doi.org/10.1016/j.cognition.2016.10.014

Bago, B., \& De Neys, W. (2019). The Smart System 1: Evidence for the intuitive nature of correct responding on the bat-and-ball problem. Thinking and Reasoning. https://doi.org/10.1080/13546783.2018.1507949

Bago, B., \& De Neys, W. (2020). Advancing the specification of dual process models of higher cognition: A critical test of the hybrid model view. Thinking and Reasoning. https://doi.org/10.1080/13546783.2018.1552194

Bago, B., Raoelison, M., \& De Neys, W. (2019). Second-guess: Testing the specificity of error detection in the bat-and-ball problem. Acta Psychologica. https://doi.org/10.1016/j.actpsy.2019.01.008

Barr, D. J., Levy, R., Scheepers, C., \& Tily, H. J. (2013). Random effects structure for confirmatory hypothesis testing: Keep it maximal. Journal of Memory and Language. https://doi.org/10.1016/j.jml.2012.11.001

Bolker, B. M., Brooks, M. E., Clark, C. J., Geange, S. W., Poulsen, J. R., Stevens, M. H. H., \& White, J.-S. S. (2009). Generalized linear mixed models: A practical guide for ecology and evolution. Trends in Ecology \& Evolution, 24(3), 127-135.

Bonnefond, M., Van der Henst, J.-B., Gougain, M., Robic, S., Olsen, M. D., Weiss, O., \& Noveck, I. (2012). How pragmatic interpretations arise from conditionals: Profiling the Affirmation of the 
Consequent argument with reaction time and EEG measures. Journal of Memory and Language, 67(4), 468-485.

Braine, M. D., \& O'Brien, D. P. (1998). The theory of mental-propositional logic: Description and illustration.

Bürkner, P.-C. (2017). brms: An R package for Bayesian multilevel models using Stan. Journal of Statistical Software, 80(1), 1-28.

Chater, N., \& Oaksford, M. (1999). The probability heuristics model of syllogistic reasoning. Cognitive Psychology, 38(2), 191-258.

De Neys, W. (2012). Bias and conflict: A case for logical intuitions. Perspectives on Psychological Science, 7(1), 28-38. https://doi.org/10.1177/1745691611429354

De Neys, W. (2014). Conflict detection, dual processes, and logical intuitions: Some clarifications. Thinking and Reasoning. https://doi.org/10.1080/13546783.2013.854725

De Neys, W., \& Glumicic, T. (2008). Conflict monitoring in dual process theories of thinking. Cognition. https://doi.org/10.1016/j.cognition.2007.06.002

De Neys, W., \& Pennycook, G. (2019). Logic, fast and slow: Advances in dual-process theorizing. Current Directions in Psychological Science.

Epstein, S. (1994). Integration of the cognitive and the psychodynamic unconscious. American Psychologist. https://doi.org/10.1037/0003-066x.49.8.709

Erceg, N., Galic, Z., \& Bubić, A. (2019). Individual differences in abilities, knowledge and thinking dispositions among different types of problem solvers and their implications for the validity of reasoning tasks. https://psyarxiv.com/w5zau/

Evans, J., Clibbens, J., \& Rood, B. (1995). Bias in conditional inference: Implications for mental models and mental logic. The Quarterly Journal of Experimental Psychology, 48(3), 644-670.

Evans, J. S. B., Handley, S. J., \& Harper, C. N. (2001). Necessity, possibility and belief: A study of syllogistic reasoning. The Quarterly Journal of Experimental Psychology: Section A, 54(3), $935-958$. 
Evans, J. S. B., Handley, S. J., Neilens, H., \& Over, D. (2010). The influence of cognitive ability and instructional set on causal conditional inference. Quarterly Journal of Experimental Psychology, 63(5), 892-909.

Evans, J. S. B., Handley, S. J., Neilens, H., \& Over, D. E. (2007). Thinking about conditionals: A study of individual differences. Memory \& Cognition, 35(7), 1772-1784.

Evans, J. S. B. T. (2010). Intuition and reasoning: A dual-process perspective. Psychological Inquiry, 21(4), 313-326. https://doi.org/10.1080/1047840X.2010.521057

Evans, J. S. B. T. (2017). Dual process theory: Perspectives and problems. In Dual Process Theory 2.0. https://doi.org/10.4324/9781315204550

Evans, J. S. B. T., \& Over, D. E. (2004). If: Supposition, pragmatics, and dual processes. Oxford University Press, USA.

Evans, J. S. B. T., \& Stanovich, K. E. (2013). Dual-Process Theories of Higher Cognition: Advancing the Debate. Perspectives on Psychological Science. https://doi.org/10.1177/1745691612460685

Evans, J. S. B. T., Thompson, V. A., \& Over, D. E. (2015). Uncertain deduction and conditional reasoning. Frontiers in Psychology. https://doi.org/10.3389/fpsyg.2015.00398

Evans, J. St. B. T. (2008). Dual-Processing Accounts of Reasoning, Judgment, and Social Cognition. Annual Review of Psychology. https://doi.org/10.1146/annurev.psych.59.103006.093629

Evans, J. St. B. T., Handley, S. J., Harper, C. N., \& Johnson-Laird, P. (1999). Reasoning about necessity and possibility: A test of the mental model theory of deduction. Journal of Experimental Psychology: Learning, Memory, and Cognition, 25(6), 1495.

Frederick, S. (2005). Cognitive reflection and decision making. In Journal of Economic Perspectives. https://doi.org/10.1257/089533005775196732

Frey, D., Johnson, E. D., \& De Neys, W. (2017). Individual differences in conflict detection during reasoning. The Quarterly Journal of Experimental Psychology, 1-52. https://doi.org/10.1080/17470218.2017.1313283 
Ghasemi, O., Handley, S., \& Howarth, S. (2021). The bright homunculus in our head: Individual differences in intuitive sensitivity to logical validity. Quarterly Journal of Experimental Psychology (2006), 17470218211044692. https://doi.org/10.1177/17470218211044691

Ghasemi, O., Handley, S., Howarth, S., Newman, I. R., \& Thompson, V. (2021). Logical Intuition Is Not Really About Logic [Data set]. OSF. https://doi.org/10.17605/OSF.IO/MFHSQ

Handley, S. J., Newstead, S. E., \& Trippas, D. (2011). Logic, Beliefs, and Instruction: A Test of the Default Interventionist Account of Belief Bias. Journal of Experimental Psychology: Learning Memory and Cognition, 37(1), 28-43. https://doi.org/10.1037/a0021098

Handley, S. J., \& Trippas, D. (2015). Dual processes and the interplay between knowledge and structure: A new parallel processing model. Psychology of Learning and Motivation - Advances in Research and Theory. https://doi.org/10.1016/bs.plm.2014.09.002

Handley, S. J., Verde, M. F., \& Morsanyi, K. (2016). Logic brightens my day: Evidence for implicit sensitivity to logical validity. Journal of Experimental Psychology: Learning Memory and Cognition, 42(9), 1448-1457. https://doi.org/10.1037/xlm0000248

Hayes, B. K., Wei, P., Dunn, J. C., \& Stephens, R. G. (2020). Why is logic so likeable? A single-process account of argument evaluation with logic and liking judgments. Journal of Experimental Psychology: Learning, Memory, and Cognition. https://doi.org/10.1037/xlm0000753

Heim, A. (1970). Manual for the AH4 group test of general intelligence. Windsor: NFER.

Howarth, S., Handley, S. J., \& Walsh, C. (2016). The logic-bias effect: The role of effortful processing in the resolution of belief-logic conflict. Memory and Cognition. https://doi.org/10.3758/s13421-015-0555-x

Howarth, S., Handley, S., \& Polito, V. (2021). Uncontrolled logic: Intuitive sensitivity to logical structure in random responding. Thinking \& Reasoning, 1-36.

Howarth, S., Handley, S., \& Walsh, C. (2018). The logic sense: Exploring the role of executive functioning in belief and logic-based judgments. Thinking \& Reasoning, 1-33. https://doi.org/10.1080/13546783.2018.1523808

Johnson-Laird, P., \& Byrne, R. M. (1991). Deduction. Lawrence Erlbaum Associates, Inc. 
Johnson-Laird, P., \& Byrne, R. M. (2002). Conditionals: A theory of meaning, pragmatics, and inference. Psychological Review, 109(4), 646.

Kahneman, D., \& Frederick, S. (2002). Representativeness revisitet. Heuristics of Intuitive Judgment: Extensions and Applications. https://doi.org/10.1038/2251090a0

Klauer, K. C., Musch, J., \& Naumer, B. (2000). On belief bias in syllogistic reasoning. Psychological Review. https://doi.org/10.1037/0033-295X.107.4.852

Klauer, K. C., \& Singmann, H. (2013). Does logic feel good? Testing for intuitive detection of logicality in syllogistic reasoning. Journal of Experimental Psychology: Learning Memory and Cognition. https://doi.org/10.1037/a0030530

Kruschke, J. (2014). Doing Bayesian data analysis: A tutorial with R, JAGS, and Stan.

Kurz, A. S. (2021). Doing Bayesian Data Analysis in brms and the tidyverse. https://bookdown.org/content/3686/

Lenth, R., Singmann, H., Love, J., Buerkner, P., \& Herve, M. (2018). Package ‘emmeans'. In R package version 1.15-15. https://doi.org/10.1080/00031305.1980.10483031>.License

Markovits, H., de Chantal, P.-L., Brisson, J., Dubé, É., Thompson, V., \& Newman, I. (2020). Reasoning strategies predict use of very fast logical reasoning. Memory \& Cognition, 1-12.

Markovits, H., \& Potvin, F. (2001). Suppression of valid inferences and knowledge structures: The curious effect of producing alternative antecedents on reasoning with causal conditionals. Memory \& Cognition, 29(5), 736-744.

McCabe, C., Halvorson, M. A., King, K., Cao, X., \& Kim, D. (2020). Estimating and interpreting interaction effects in generalized linear models of binary and count data.

Mevel, K., Poirel, N., Rossi, S., Cassotti, M., Simon, G., Houdé, O., \& De Neys, W. (2015). Bias detection: Response confidence evidence for conflict sensitivity in the ratio bias task. Journal of Cognitive Psychology. https://doi.org/10.1080/20445911.2014.986487

Mize, T. D. (2019). Best practices for estimating, interpreting, and presenting nonlinear interaction effects. Sociological Science, 6, 81-117.

Morewedge, C. K., \& Kahneman, D. (2010). Associative processes in intuitive judgment. In Trends in Cognitive Sciences. https://doi.org/10.1016/j.tics.2010.07.004 
Morsanyi, K., \& Handley, S. J. (2008). How smart do you need to be to get it wrong? The role of cognitive capacity in the development of heuristic-based judgment. Journal of Experimental Child Psychology, 99(1), 18-36.

Morsanyi, K., \& Handley, S. J. (2012). Logic feels so good-I like it! Evidence for intuitive detection of logicality in syllogistic reasoning. Journal of Experimental Psychology: Learning Memory and Cognition. https://doi.org/10.1037/a0026099

Nakamura, H., \& Kawaguchi, J. (2016). People like logical truth: Testing the intuitive detection of logical value in basic propositions. PLoS ONE. https://doi.org/10.1371/journal.pone.0169166

Newman, I. R., Gibb, M., \& Thompson, V. A. (2017). Rule-based reasoning is fast and belief-based reasoning can be slow: Challenging current explanations of belief-bias and base-rate neglect. Journal of Experimental Psychology: Learning Memory and Cognition. https://doi.org/10.1037/xlm0000372

Newstead, S. E., Handley, S. J., Harley, C., Wright, H., \& Farrelly, D. (2004). Individual differences in deductive reasoning. The Quarterly Journal of Experimental Psychology. A, Human Experimental Psychology. https://doi.org/10.1080/02724980343000116

Newstead, S. E., Pollard, P., Evans, J. S. B. T., \& Allen, J. L. (1992). The source of belief bias effects in syllogistic reasoning. Cognition. https://doi.org/10.1016/0010-0277(92)90019-E

Peirce, J., Gray, J. R., Simpson, S., MacAskill, M., Höchenberger, R., Sogo, H., Kastman, E., \& Lindeløv, J. K. (2019). PsychoPy2: Experiments in behavior made easy. Behavior Research Methods. https://doi.org/10.3758/s13428-018-01193-y

Pennycook, G., Fugelsang, J. A., \& Koehler, D. J. (2015). What makes us think? A three-stage dualprocess model of analytic engagement. Cognitive Psychology. https://doi.org/10.1016/j.cogpsych.2015.05.001

Pennycook, G., Neys, W. D., Evans, J. S. B. T., Stanovich, K. E., \& Thompson, V. A. (2018). The Mythical Dual-Process Typology. In Trends in Cognitive Sciences. https://doi.org/10.1016/j.tics.2018.04.008 
Pennycook, G., Trippas, D., Handley, S. J., \& Thompson, V. A. (2014). Base rates: Both neglected and intuitive. Journal of Experimental Psychology: Learning Memory and Cognition, 40(2), 544554. https://doi.org/10.1037/a0034887

R Core Team. (2014). R: A language and environment for statistical computing. $R$ Foundation for Statistical Computing, Vienna, Austria. URL Http://Www.R-Project.Org/.

Rader, A. W., \& Sloutsky, V. M. (2002). Processing of logically valid and logically invalid conditional inferences in discourse comprehension. Journal of Experimental Psychology: Learning, Memory, and Cognition, 28(1), 59.

Raoelison, M., Boissin, E., Borst, G., \& De Neys, W. (2021). From slow to fast logic: The development of logical intuitions. Thinking \& Reasoning, 1-25.

Raoelison, M., Thompson, V. A., \& De Neys, W. (2020). The smart intuitor: Cognitive capacity predicts intuitive rather than deliberate thinking. Cognition. https://doi.org/10.1016/j.cognition.2020.104381

Ricco, R. B., Koshino, H., Sierra, A. N., Bonsel, J., Monteza, J. V., \& Owens, D. (2020). Individual differences in analytical thinking and complexity of inference in conditional reasoning. Thinking and Reasoning. https://doi.org/10.1080/13546783.2020.1794958

Rips, L. J. (1994). The psychology of proof: Deductive reasoning in human thinking. Mit Press.

Rumain, B., Connell, J., \& Braine, M. D. (1983). Conversational comprehension processes are responsible for reasoning fallacies in children as well as adults: If is not the biconditional. Developmental Psychology, 19(4), 471.

Schaeken, W., \& Schroyens, W. (2000). The effect of explicit negatives and of different contrast classes on conditional syllogisms. British Journal of Psychology, 91(4), 533-550.

Schroyens, W., \& Braem, S. (2011). The initial representation in reasoning towards an interpretation of conditional sentences. Quarterly Journal of Experimental Psychology, 64(2), 339-362.

Schroyens, W., Schaeken, W., \& d'Ydewalle, G. (2001). The processing of negations in conditional reasoning: A meta-analytic case study in mental model and/or mental logic theory. Thinking \& Reasoning, 7(2), 121-172. 
Schroyens, W., Schaeken, W., Fias, W., \& d'Ydewalle, G. (2000). Heuristic and analytic processes in propositional reasoning with negatives. Journal of Experimental Psychology: Learning, Memory, and Cognition, 26(6), 1713.

Schroyens, W., Schaeken, W., \& Handley, S. (2003). In search of counter-examples: Deductive rationality in human reasoning. The Quarterly Journal of Experimental Psychology Section A, 56(7), 1129-1145.

Singmann, H., Bolker, B., Westfall, J., Aust, F., \& Ben-Shachar, M. S. (2015). afex: Analysis of factorial experiments. $R$ Package Version 0.13-145.

Singmann, H., \& Kellen, D. (2019). An Introduction to Mixed Models for Experimental Psychology. In New Methods in Cognitive Psychology. https://doi.org/10.4324/9780429318405-2

Singmann, H., Klauer, K. C., \& Beller, S. (2016). Probabilistic conditional reasoning: Disentangling form and content with the dual-source model. Cognitive Psychology. https://doi.org/10.1016/j.cogpsych.2016.06.005

Singmann, H., Klauer, K. C., \& Kellen, D. (2014). Intuitive logic revisited: New data and a bayesian mixed model meta-analysis. PLoS ONE, 9(4). https://doi.org/10.1371/journal.pone.0094223

Sloman, S. A. (1996). The empirical case for two systems of reasoning. Psychological Bulletin. https://doi.org/10.1037/0033-2909.119.1.3

Sloman, S. A. (2012). Two Systems of Reasoning. In Heuristics and Biases. https://doi.org/10.1017/cbo9780511808098.024

Šrol, J., \& De Neys, W. (2020). Predicting individual differences in conflict detection and bias susceptibility during reasoning. Thinking and Reasoning. https://doi.org/10.1080/13546783.2019.1708793

Stanovich, K. E. (2018). Miserliness in human cognition: The interaction of detection, override and mindware. Thinking \& Reasoning. https://doi.org/10.1080/13546783.2018.1459314

Thompson, V. A. (1994). Interpretational factors in conditional reasoning. Memory \& Cognition, 22(6), $742-758$.

Thompson, V. A., \& Johnson, S. C. (2014). Conflict, metacognition, and analytic thinking. Thinking and Reasoning. https://doi.org/10.1080/13546783.2013.869763 
Thompson, V. A., \& Newman, I. R. (2017). Logical intuitions and other conundra for dual process theories. In Dual Process Theory 2.0. https://doi.org/10.4324/9781315204550

Thompson, V. A., Pennycook, G., Trippas, D., \& Evans, J. S. B. T. (2018). Do smart people have better intuitions? Journal of Experimental Psychology: General, 147(7), 945-961. https://doi.org/10.1037/xge0000457

Thompson, V. A., Prowse Turner, J. A., \& Pennycook, G. (2011). Intuition, reason, and metacognition. Cognitive Psychology, 63(3), 107-140. https://doi.org/10.1016/j.cogpsych.2011.06.001

Toplak, M. E., West, R. F., \& Stanovich, K. E. (2011). The Cognitive Reflection Test as a predictor of performance on heuristics-and-biases tasks. Memory and Cognition, 39(7), 1275-1289. https://doi.org/10.3758/s13421-011-0104-1

Toplak, M. E., West, R. F., \& Stanovich, K. E. (2014). Assessing miserly information processing: An expansion of the Cognitive Reflection Test. Thinking and Reasoning. https://doi.org/10.1080/13546783.2013.844729

Topolinski, S. (2018). The sense of coherence: How intuition guides reasoning and thinking. https://psycnet.apa.org/record/2017-56397-031

Topolinski, S., \& Reber, R. (2010). Immediate truth-Temporal contiguity between a cognitive problem and its solution determines experienced veracity of the solution. Cognition. https://doi.org/10.1016/j.cognition.2009.09.009

Trippas, D., \& Handley, S. J. (2018). The Parallel Processing Model of Belief Bias. In Dual Process Theory 2.0 (pp. 28-46). https://doi.org/10.4324/9781315204550-3

Trippas, D., Handley, S. J., Verde, M. F., \& Morsanyi, K. (2016). Logic brightens my day: Evidence for implicit sensitivity to logical validity. Journal of Experimental Psychology: Learning Memory and Cognition. https://doi.org/10.1037/xlm0000248

Trippas, D., Handley, S. J., Verde, M. F., van Tiel, B., \& Verde, M. F. (2013). The SDT model of belief bias: Complexity, time, and cognitive ability mediate the effects of believability. Journal of Experimental Psychology: Learning Memory and Cognition, 39(5), 1393-1402. https://doi.org/10.1037/a0032398 
Trippas, D., Thompson, V. A., \& Handley, S. J. (2017). When fast logic meets slow belief: Evidence for a parallel-processing model of belief bias. Memory and Cognition, 45(4), 539-552. https://doi.org/10.3758/s13421-016-0680-1

Tversky, A., \& Kahneman, D. (1983). Extensional versus intuitive reasoning: The conjunction fallacy in probability judgment. Psychological Review. https://doi.org/10.1037/0033-295X.90.4.293

Vehtari, A., Gelman, A., \& Gabry, J. (2017). Practical Bayesian model evaluation using leave-one-out cross-validation and WAIC. Statistics and Computing, 27(5), 1413-1432.

Wetherick, N. E., \& Gilhooly, K. J. (1995). 'Atmosphere', matching, and logic in syllogistic reasoning. Current Psychology, 14(3), 169-178. 